Article

\title{
Analysis of Decision Support System Based on 2-Tuple Spherical Fuzzy Linguistic Aggregation Information
}

\author{
Saleem Abdullah ${ }^{1, *(\mathbb{D}, \text { Omar Barukab }}{ }^{2}$, Muhammad Qiyas ${ }^{1}\left(\mathbb{D}\right.$, Muhammad Arif $^{1}(\mathbb{D}$ and \\ Sher Afzal Khan ${ }^{1}$ \\ 1 Faculty of Physical and Numerical Sciences, Abdul Wali Khan University Mardan, Mardan 23200, Pakistan; \\ muhammadqiyas@awkum.edu.pk (M.Q.); marifmaths@awkum.edu.pk (M.A.); \\ Sher.afzal@awkum.edu.pk (S.A.K.) \\ 2 Faculty of Computing and Information Technology, King Abdulaziz University, P.O. Box 411, Rabigh 21911, \\ Jeddah, Saudi Arabia; obarukab@kau.edu.sa \\ * Correspondence: saleemabdullah@awkum.edu.pk
}

Received: 27 October 2019; Accepted: 10 December 2019; Published: 30 December 2019

check for updates

\begin{abstract}
The aim of this paper is to propose the 2-tuple spherical fuzzy linguistic aggregation operators and a decision-making approach to deal with uncertainties in the form of 2-tuple spherical fuzzy linguistic sets. 2-tuple spherical fuzzy linguistic operators have more flexibility than general fuzzy set. We proposed a numbers of aggregation operators, namely 2-tuple spherical fuzzy linguistic weighted average, 2-tuple spherical fuzzy linguistic ordered weighted average, 2-tuple spherical fuzzy linguistic hybrid average, 2-tuple spherical fuzzy linguistic weighted geometric, 2-tuple spherical fuzzy linguistic ordered geometric, and 2-tuple spherical fuzzy linguistic hybrid geometric operators. The distinguishing feature of these proposed operators is studied. At that point, we have used these operators to design a model to deal with multiple attribute decision-making issues under the 2-tuple spherical fuzzy linguistic information. Then, a practical application for best company selection for feeds is given to prove the introduced technique and to show its practicability and effectiveness. Besides this, a systematic comparison analysis with other existent methods is conducted to reveal the advantage of our method. Results indicate that the proposed method is suitable and effective for decision making problems.
\end{abstract}

Keywords: spherical fuzzy set; 2-tuple linguistic model; 2-tuple spherical fuzzy linguistic set

\section{Introduction}

Universally, in the representation of data information there exists some uncertainty in the decision-making (DM) process. To overcome this drawback, first time Zadeh's [1] defined fuzzy set (FS). In FS, Zadeh's only show the positive membership grade of a number in the defined set, and applied to many fields i.e., fuzzy decision making problems [2,3]. However, there are no discussion for the negative membership grade. Then, the fuzzy set theory failed to solve the complete uncertainty in the real life problem due to non-membership grade. So, Atanassov [4] defined an intuitionistic fuzzy set (IFS) concept, which represents positive and negative membership grades; IFS has the advantage of two memberships which diminish the fuzziness. Garg [5,6] displayed generalized improved interactive aggregation operators for the solution of a decision-making problem with the IF set condition. Kaur and Garg [7] introduced a cubic intuitionistic fuzzy set (CIFS) aggregation operator. Later, Kaur \& Garg [8] developed generalized aggregation operators with the cubic IF set information using the t-norm operations. Aside from them, different authors (Garg \& Arora [9]; Garg \& Rani [10]; Shen \& Wang [11]; Wang, Peng, \& Wang [12]) incorporated the idea of aggregation process into the 
different applications and also give their related decision-making approaches with the IF set and their expansion. Garg \& Kumar [13] developed the power geometric aggregation operators using the concept of connection number of set pair analysis theory with intuitionistic fuzzy information. Garg \& Rani [14] proposed a new generalized Bonferroni mean aggregation operators of complex intuitionistic fuzzy information based on Archimedean t-norm and t-conorm. Garg \& Arora [15] introduced the generalized intuitionistic fuzzy soft power aggregation operator using the $\mathrm{t}$-norm and its application in multi-criteria decision-making.

There are some situations, where $\mu+v \geq 1$, unlike the cases captured in IFSs. Then, the Pythagorean fuzzy set (PFS) is defined by Yager $[16,17]$, which is characterized by positive and negative membership grades, and satisfies that the square of the positive and negative membership grades and their sum is less than or equal to 1. To handle these type of situation Yager [17] gives an example: a decision maker provides his positive suggestion for an alternative is $\frac{\sqrt{3}}{2}$ and his negative suggestion is $\frac{1}{2}$. Now, their sum is greater than 1 , then they are not possible for IF set, but they are possible for PFS, because $\left(\frac{\sqrt{3}}{2}\right)^{2}+\left(\frac{1}{2}\right)^{2} \leq 1$. This shows that PFS is more able than IF set to process the uncertainty in real life problems. In recent times, the notion of PF set invites the thinking of researchers, who have applied the PF set in many research areas. Rahman et al. [18] introduced the geometric aggregation operators for the group decision-making problem with interval valued Pythagorean fuzzy sets (IVPFSs) environment.Liang \& Xu [19] proposed the notion of hesitant PF sets, and applied The Technique for Order of Preference by Similarity to Ideal Solution (TOPSIS) method for energy project selection model. Rahman et al. [20] introduced a new algorithm for the multi-attribute group decision making (MAGDM) problem, using induced IVPF Einstein aggregation operator. Garg [21] defined a decision-making model with probabilistic information, utilizing the notion of immediate probabilities to aggregate the data with the PFS information. Utilizing the notion of the immediate probabilities, Garg [22] introduced a series of the aggregation operators with the PF information. Garg [23] developed the generalized PF geometric aggregation operators utilizing different norm operations for multi-attribute group decision-making problems. Ren et al. [24] introduced TODIM technique for finding the best alternative in decision making problems, where the information occurred in the form of PF numbers. Wei [25] introduced many aggregation operators such as interaction weighted averaging and weighted geometric operators. Using PF information, Wei \& $\mathrm{Lu}$ [26] developed the power aggregation operators to deal with an MAGDM problem. Xu et al. [27] launched the induced generalized OWA operators for PF information. Xue et al. [28] developed the LINMAP technique to track the best investment company in railway projects using PF information. Perez-Dominguez [29] developed a multi-objective optimization based on ratio analysis (MOORA) with PF set information and applied it to MAGDM problem. To solve an MAGDM problem with partially known weight information, Garg [30] introduced an improved score function, where the preferences of the attribute are taken in the form of IVPF sets. Hamacher operations [31], including Hamacher product and sum, are good alternatives to the algebraic product and sum, respectively [32]. In last few years, many researchers discussed the Hamacher aggregation operators and their applications [33,34].

Herrera \& Martinez [35] developed the concept of 2-tuple linguistic processing model based on the symbolic translation model, and also showed that the 2-tuple linguistic information processing manner can effectively avoid the loss and distortion of information. A group decision-making model was proposed by Herrera et al. [36], to manage a non homogeneous information. For choosing the appropriate agile manufacturing system, Wang [37] developed a 2-tuple fuzzy linguistic processing model. The TOPSIS technique are extended by Wei [38] for MAGDM problem with 2-tuple linguistic information. Chang \& Wen [39] proposed the efficient algorithm for DFMEA combining 2-tuple and the OWA operator. Bonferroni mean operators are extended by Jiang \& Wei [40] for the 2-tuple linguistic information. The dependent interval 2-tuple linguistic aggregation operators are proposed by Liu et al. [41] for MAGDM. Wang et al. [42] proposed an algorithm for the MAGDM problems, using the interval 2-tuple linguistic information and Choquet integral aggregation operators. To study the application of MAGDM on the supplier selection Liu [43] defined the 2-tuple linguistic Muirhead 
mean operators. A consensus-reaching model for the 2-tuple linguistic MAGDM was proposed by Zhang et al. [44], where the weight information was incomplete.

The notion of spherical fuzz set (SFS) was first time introduced by Ashraf et al. [45], and defined some aggregation operators for MADM problem with the spherical fuzzy information. Gundogdu et al. [46] defined the extended TOPSIS method to spherical fuzzy TOPSIS and an illustrative example is presented for multi-criteria decision making problem. Basically, spherical fuzzy set is the extended form of picture fuzzy set and Pythagorean fuzzy set. In the SF set, all the membership grades are satisfying the condition $0 \leq\left(\mu_{\Re}(r)\right)^{2}+\left(\eta_{\Re}(r)\right)^{2}+\left(\nu_{\Re}(r)\right)^{2} \leq 1$ instead of $0 \leq \mu_{\Re}(r)+\eta_{\Re}(r)+v_{\Re}(r) \leq 1$ as in picture fuzzy set. Huanhuan et al. [47] defined linguistic spherical fuzzy set, combining the idea of linguistic fuzzy set and spherical fuzzy set. Shahzaib et al. [48] defined some spherical fuzzy aggregation operators, using Dombi operation, discussed their application on decision making, and also discussed the representation of spherical fuzzy t-norm and t-conorm in [49].

Due to the motivation and inspiration of the above discussion in this article we introduce the notion of 2-tuple spherical fuzzy linguistic sets, each element of which is composed of linguistic postive, neutral, and negative membership grades. We introduce the aggregation techniques for 2-tuple spherical fuzzy linguistic numbers. First, we define the concept of 2-tuple spherical fuzzy linguistic numbers (2TSFLNs), then introduce some basic operational laws of 2TSFLNs. We define the score function of 2TSFLNs, based on which a simple method for ranking the 2TSFLNs is presented. We also introduce a number of operators for aggregating 2TSFLNs, including the averaging operators and geometric operators and established various properties of these operators. Some approaches to multi-attribute decision making based on 2TSFLNs are presented, and applied these approachs in the field of decision making.

For this purpose, the reminder of the article is structured as; In Section 2, we briefly discuss the basic knowledge about the 2-tuple linguistic model and spherical fuzzy set. In Section 3, we develop the concept of 2-tuple spherical fuzzy linguistic set. In Section 4, we present some 2-tuple spherical fuzzy linguistic averaging aggregation (2TSFLWA) operators, and discussed some basic properties of the proposed operators. In Section 5, we present some 2-tuple spherical fuzzy linguistic geometric aggregation (2TSFLWG) operators, and discussed some basic properties of the proposed operators. In Section 6, we use the 2TSFLWA and 2TSFLWG operators, and proposed an algorithm for multi-attribute decision-making problem, and makes some discussions on application of developed technique. In Section 7, we discuss their comparison with existing approachs, and finally write the conclusion of the paper in Section 8.

\section{Preliminaries}

In this section, we present basic knowledge about the 2-tuple linguistic term set, spherical fuzzy set, and 2-tuple spherical fuzzy linguistic set.

Definition 1 ([35]). Let $\dot{S}=\left(\hat{s}_{1}, \ldots, \kappa\right)$ be the collection of linguistic term, and $\kappa$ denotes the odd cardinality, such that $\dot{s}_{\kappa}$, and $\kappa$ represent the possible value of the linguistic variable and positive integer, respectively. Generally, $\kappa$ is taken as 3,5, etc. For example, when $\kappa=5$, then the linguistic term set $S$ is defined as $\left\{\hat{s}_{1}=\right.$ poor, $\hat{s}_{2}=$ slightly poor, $\hat{s}_{3}=$ fair, $\dot{s}_{4}=$ slightly good,$\hat{s}_{5}=$ good $\}$.

If $\hat{s}_{\kappa}, \hat{s}_{t} \in \dot{S}$, then the following characteristic must be satisfied;

(1) The ordered of set as: $\hat{s}_{\kappa} \prec \dot{s}_{t}, \Leftrightarrow \kappa \prec t$;

(2) The operator of negation as: Neg $\left(\hat{s}_{\kappa}\right)=\hat{s}_{\kappa-\kappa}$;

(3) $\operatorname{Maximum}\left(\hat{s}_{\kappa}, \hat{s}_{t}\right)=\hat{s}_{\kappa}$, iff $\hat{s}_{\kappa} \geq \hat{s}_{t}$;

(4) $\operatorname{Minimum}\left(\hat{s}_{\kappa}, \hat{s}_{t}\right)=\hat{s}_{\kappa}$, iff $\hat{s}_{\kappa} \leq \hat{s}_{t}$.

Using the concept of symbolic translation, Herrera and Martinez [50,51] introduced the 2-tuple fuzzy linguistic model. This model is used to denote the linguistic assessment information by means of a 2-tuple $\left(\hat{s}_{l}, \ddot{a}_{l}\right)$, and $\hat{s}_{l}$ and $\ddot{a}_{l}$ denote the linguistic label and symbolic translation respectively, from the linguistic term set $S$ and $\ddot{a} \in[-0.5,0.5]$. 
Definition 2 ([35]). Let ë be the result of an aggregation of the indices of a set of labels assessed in a linguistic term set $\dot{S}$, for example the result of a symbolic aggregation operation, $\ddot{e} \in[1, \kappa]$, where $\kappa$ denote the odd cardinality of $\dot{S}$. Let $\imath=\operatorname{round}(\ddot{e})$ and $\ddot{a}=\ddot{e}-\imath$ be two values, such that, $\imath \in[1, \kappa]$ and $\ddot{a} \in[-0.5,0.5]$, then $\ddot{a}$ is said to be the symbolic translation.

Definition 3 ([35]). Let $\hat{S}=\left(\hat{s}_{1}, \ldots, \kappa\right)$ be the finite linguistic term set and $\ddot{e} \in[1, \kappa]$ be the number value of the aggregation result of linguistic symbolic. Then, the mapping $\Xi$ is utilized to get the 2-tuple linguistic information equivalent to $\ddot{e}$, and is defined as:

$$
\begin{gathered}
\Xi:[1, \kappa] \rightarrow \dot{S} \times[-0.5,0.5), \\
\Xi(\ddot{e})=\left\{\begin{array}{c}
\dot{s}_{l}, \imath=\operatorname{round}(\ddot{e}) \\
\ddot{a}=\ddot{e}-\imath, \ddot{a} \in[-0.5,0.5),
\end{array}\right.
\end{gathered}
$$

where round $(),. \hat{s}_{l}, \ddot{a}$ denotes the usual round operation, closest index label to $\ddot{e}$, and the value of the symbolic translation, respectively.

Definition 4 ([35]). Let $\hat{S}=\left(\hat{s}_{1}, \ldots, \kappa\right)$ be the finite linguistic term set and $\left(\hat{s}_{1}, \ddot{a}_{1}\right)$ be a 2-tuple. Then, there exists a mapping $\Xi^{-1}$, such that from a 2-tuple $\left(\hat{s}_{l}, \ddot{a}_{l}\right)$ it returns its equivalent numerical value $\ddot{e} \in[1, \kappa] \subset R$, which is;

$$
\begin{gathered}
\Xi^{-1}: \hat{S} \times[-0.5,0.5) \rightarrow[1, \kappa], \\
\Xi^{-1}\left(\hat{s}_{\imath}, \ddot{a}\right)=\imath+\ddot{a}=\ddot{e},
\end{gathered}
$$

From Definitions 2 and 3, we observe that the conversion of a linguistic term into a linguistic 2-tuple consists of adding a value 0 as symbolic translation:

$$
\Xi\left(\dot{s}_{l}\right)=\left(\dot{s}_{l}, 0\right)
$$

Definition 5 ([46]). Let $\mathbb{R} \neq \phi$, be a set. Then, $\Re$ is called spherical fuzzy set, and defined as;

$$
\Re=\left\{\left\langle\mu_{\Re}(r), \eta_{\Re}(r), \nu_{\Re}(r) \mid r \in \mathbb{R}\right\rangle\right\} .
$$

where $\mu_{\Re}(r), \eta_{\Re}(r), \nu_{\Re}(r): \mathbb{R} \rightarrow[0,1]$ are the positive, neutral, and negative membership grades of each $r \in \mathbb{R}$, correspondingly. Furthermore, $\mu_{\Re}(r), \eta_{\Re}(r)$ and $v_{\Re}(r)$ satisfy that $0 \leq \mu_{\Re}^{2}(r)+\eta_{\Re}^{2}(r)+v_{\Re}^{2}(r) \leq 1 \forall r \in \mathbb{R}$. $\pi_{\Re}(r)=\sqrt{1-\left(\mu_{\Re}^{2}(r)+\eta_{\Re}^{2}(r)+v_{\Re}^{2}(r)\right)}$ is said to be a refusal grade of $r$ in $\mathbb{R}$, and a triple components $\left\langle\mu_{\Re}, \eta_{\Re}, \nu_{\Re}\right\rangle$ are called the SF number and each SF number is denoted by $\Re=\left\langle\mu_{\Re}, \eta_{\Re}, \nu_{\Re}\right\rangle$, where $\mu_{\Re,} \eta_{\Re}$, and $\nu_{\Re} \in[0,1]$, have the condition

$$
0 \leq \mu_{\Re}^{2}+\eta_{\Re}^{2}+v_{\Re}^{2} \leq 1 .
$$

Definition 6 ([46]). Let $\Re_{1}=\left\langle\mu_{\Re_{1}}(r), \eta_{\Re_{1}}(r), v_{\Re_{1}}(r)\right\rangle$, and $\Re_{2}=\left\langle\mu_{\Re_{2}}(r), \eta_{\Re_{2}}(r), v_{\Re_{2}}(r)\right\rangle$ be two SFNs defined on the universe of discourse $\mathbb{R} \neq \phi$, some operations on SFNs are defined as follows:

(1) $\Re_{1} \subseteq \Re_{2}$ if

$$
\mu_{\Re_{1}}^{2}(r) \leq \mu_{\Re_{2}}^{2}(r), \eta_{\Re_{1}}^{2}(r) \leq \eta_{\Re_{2}}^{2}(r) \text { and } v_{\Re_{1}}^{2}(r) \geq v_{\Re_{2}}^{2}(r), \forall r \in \mathbb{R},
$$

(2) Union

$$
\Re_{1} \cup \Re_{2}=\left(\begin{array}{c}
\left(r, \max \left(\mu_{\Re_{1}}^{2}(r), \mu_{\Re_{2}}^{2}(r)\right), \min \left(\eta_{\Re_{1}}^{2}(r), \eta_{\Re_{2}}^{2}(r)\right),\right. \\
\min \left(v_{\Re_{1}}^{2}(r), v_{\Re_{2}}^{2}(r)\right) \mid r \in \mathbb{R}
\end{array}\right) ;
$$


(3) Intersection

$$
\Re_{1} \cap \Re_{2}=\left(\begin{array}{c}
\left(r, \min \left(\mu_{\Re_{1}}^{2}(r), \mu_{\Re_{2}}^{2}(r)\right), \min \left(\eta_{\Re_{1}}^{2}(r), \eta_{\Re_{2}}^{2}(r)\right),\right. \\
\max \left(v_{\Re_{1}}^{2}(r), v_{\Re_{2}}^{2}(r)\right) \mid r \in \mathbb{R}
\end{array}\right) ;
$$

(4) Compliment

$$
\Re_{1}^{c}=\left\{\left(r, v_{\Re_{1}}^{2}(r), \eta_{\Re_{1}}^{2}(r), \mu_{\Re_{1}}^{2}(r) \mid r \in \mathbb{R}\right\} .\right.
$$

Definition 7 ([46]). Let $\Re_{1}=\left\langle\mu_{\Re_{1}}, \eta_{\Re_{1}}, v_{\Re_{1}}\right\rangle$ and $\Re_{2}=\left\langle\mu_{\Re_{2}}, \eta_{\Re_{2}}, \nu_{\Re_{2}}\right\rangle$ be two SFNs defined on the universe of discourse $\mathbb{R} \neq \alpha$, some operations on SFNs are defined as follows with $\lambda \geq 0$.

1. $\Re_{1} \oplus \Re_{2}=\left\{\sqrt{\mu_{\Re_{1}}^{2}+\mu_{\Re_{2}}^{2}-\mu_{\Re_{1}}^{2} \cdot \mu_{\Re_{2}}^{2}}, \eta_{\Re_{1}} \cdot \eta_{\Re_{2}}, v_{\Re_{1}} \cdot \nu_{\Re_{2}}\right\} ;$

2. $\Re_{1} \otimes \Re_{2}=\left\{\mu_{\Re_{1}} \cdot \mu_{\Re_{2}}, \sqrt{\eta_{\Re_{1}}^{2}+\eta_{\Re_{2}}^{2}-\eta_{\Re_{1}}^{2} \cdot \eta_{\Re_{2}}^{2}}, \sqrt{v_{\Re_{1}}^{2}+v_{\Re_{2}}^{2}-v_{\Re_{1}}^{2} \cdot v_{\Re_{2}}^{2}}\right\} ;$

3. $\lambda \otimes \Re_{1}=\left\{\sqrt{1-\left(1-\mu_{\Re_{1}}^{2}\right)^{\lambda}},\left(\eta_{\Re_{1}}\right)^{\lambda},\left(\nu_{\Re_{1}}\right)^{\lambda}\right\} ;$

4. $\Re_{1}^{\lambda}=\left\{\left(\mu_{\Re_{1}}\right)^{\lambda}, \sqrt{1-\left(1-\eta_{\Re_{1}}^{2}\right)^{\lambda}}, \sqrt{1-\left(1-v_{\Re_{1}}^{2}\right)^{\lambda}}\right\} ;$

5. $\Re_{1}^{c}=\left\langle v_{\Re_{1}}, \eta_{\Re_{1}}, \mu_{\Re_{1}}\right\rangle$;

\section{2-Tuple Spherical Fuzzy Linguistic Sets}

In the following, we introduced the concept of 2-tuple spherical fuzzy linguistic sets and their basic operations based on the spherical fuzzy set and 2-tuple linguistic information.

Definition 8. A 2-tuple spherical fuzzy linguistic set $\Re$ in $\mathbb{R} \neq \phi$, is defined as;

$$
\Re=\left\{\left\langle\hat{s}_{\mu(r)}, \hat{s}_{\eta(r)}, \hat{s}_{v(r)} \mid r \in \mathbb{R}\right\rangle\right\} .
$$

The numbers $\hat{s}_{\mu(r)}, \dot{s}_{\eta(r)}$ and $\dot{s}_{v(r)}$ are denoted the positive, neutral, and negative membership grades of the number $r$ to linguistic variable $\Re$. Where $\dot{s}_{\mu(r)}, \dot{s}_{\eta(r)}, \hat{s}_{v(r)} \in S$, with the condition $3 \leq \mu^{2}(r)+\eta^{2}(r)+v^{2}(r) \leq$ $(\kappa+1)^{2}, \forall r \in \mathbb{R}$. The term $\dot{s}_{\pi(r)}$ is called the refusal grade of the element $r$ to the set $\Re$, and defined as $\hat{s}_{\pi(r)}=\hat{s} \sqrt{(\kappa+1)^{2}-\mu^{2}-\eta^{2}-v^{2}}$.

For convenience, we say $\widetilde{\Re}=\left\langle\left(\dot{s}_{\mu(r)}, \ddot{a}\right),\left(\hat{s}_{\eta(r)}, \ddot{e}\right),\left(\dot{s}_{v(r)}, \ddot{u}\right)\right\rangle$, a 2-tuple spherical fuzzy linguistic number (2TSFLN), where $\hat{s}_{\mu(r)}, \hat{s}_{\eta(r)}, \hat{s}_{v(r)} \in S^{\prime}$,

$$
3 \leq \Xi^{-1}\left(\hat{s}_{\mu^{2}(r)}, \ddot{a}\right)+\Xi^{-1}\left(\hat{s}_{\eta^{2}(r)}, \ddot{e}\right)+\Xi^{-1}\left(\hat{s}_{v^{2}(r)}, \ddot{u}\right) \leq(\kappa+1)^{2} \text {, and } \ddot{a}, \ddot{e}, \ddot{u} \in[-0.5,0.5) \text {. }
$$

Definition 9. Let $\alpha, \beta, \gamma$ be the results of the aggregation of the indices of a set of labels assessed in a linguistic term set $S$, for example, the result of a symbolic aggregation operation, $\alpha, \beta, \gamma \in[1, \kappa], 3 \leq \alpha+\beta+\gamma \leq$ $\kappa+1$, (where $\kappa$ be the cardinality of $\dot{S})$. Assume that $\mu=\operatorname{round}(\alpha), \eta=\operatorname{round}(\beta), v=\operatorname{round}(\gamma)$ and $\ddot{a}=\alpha-\mu, \ddot{e}=\beta-\eta, \ddot{u}=\gamma-v$ are the six values, such that $\alpha, \beta, \gamma \in[1, \kappa]$, and $\ddot{a}, \ddot{e}, \ddot{u} \in[-0.5,0.5)$, then $\ddot{a}, \ddot{e}, \ddot{u}$ are called symbolic translations.

Definition 10. Let $\hat{S}=\left(\hat{s}_{1}, \ldots, \hat{s}_{\kappa}\right)$ be the linguistic set and $\alpha, \beta, \gamma \in[1, \kappa]$ be the three number value, denoting the aggregation result of linguistic symbolic. Then, a mapping $\Xi$ is utilized to get the 2-tuple linguistic information equivalent to $\alpha, \beta, \gamma$ which is defined as:

$$
\begin{gathered}
\Xi:[1, \kappa] \rightarrow \dot{S} \times[-0.5,0.5), \\
\Xi(\alpha)=\left\{\begin{array}{c}
\hat{s}_{\mu}, \mu=\operatorname{round}(\alpha) \\
\ddot{a}=\alpha-\mu, \ddot{a} \in[-0.5,0.5),
\end{array}\right.
\end{gathered}
$$




$$
\begin{aligned}
& \Xi(\beta)=\left\{\begin{array}{c}
\hat{s}_{\eta}, \eta=\operatorname{round}(\beta) \\
\ddot{e}=\beta-\eta, \ddot{e} \in[-0.5,0.5),
\end{array}\right. \\
& \Xi(\ddot{u})=\left\{\begin{array}{c}
\hat{s}_{v}, v=\operatorname{round}(\gamma) \\
\ddot{u}=\gamma-v, \ddot{u} \in[-0.5,0.5),
\end{array}\right.
\end{aligned}
$$

where round (.), $\hat{s}_{\mu}, \hat{s}_{\eta}, \hat{s}_{v}$ and $\ddot{a}, \ddot{e}, \ddot{u}$ denoted the usual round operation, closest index label to $\alpha, \beta, \gamma$ and the value of the symbolic translation, respectively.

Definition 11. Let $\dot{S}=\left(\hat{s}_{1}, \ldots, \hat{s}_{\kappa}\right)$ be the finite linguistic term set and $\widetilde{\Re}=\left\langle\left(\hat{s}_{\mu}, \ddot{a}\right),\left(\hat{s}_{\eta}, \ddot{e}\right),\left(\hat{s}_{v}, \ddot{u}\right)\right\rangle$ be a 2-tuple spherical fuzzy linguistic number (2TPFLN). Then, there exists a mapping $\Xi^{-1}$, such that from a 2-tuple spherical fuzzy numbers $\left\langle\left(\hat{s}_{\mu}, \ddot{a}\right),\left(\hat{s}_{\eta}, \ddot{e}\right),\left(\hat{s}_{v}, \ddot{u}\right)\right\rangle$ it returns its equivalent numerical value $\alpha, \beta, \gamma \in[1, \kappa] \subset R$, which is

$$
\begin{gathered}
\Xi^{-1}: \dot{S} \times[-0.5,0.5) \rightarrow[1, \kappa], \\
\Xi^{-1}\left(\dot{s}_{\mu}, \ddot{a}\right)=\mu+\ddot{a}=\alpha, \\
\Xi^{-1}\left(\hat{s}_{\eta}, \ddot{e}\right)=\eta+\ddot{e}=\beta, \\
\Xi^{-1}\left(\hat{s}_{v}, \ddot{u}\right)=v+\ddot{u}=\gamma .
\end{gathered}
$$

From Definitions 2 and 3, we observe that the conversion of a linguistic term into a linguistic 2-tuple consists of adding a value 0 as symbolic translation:

$$
\Xi\left\langle\dot{s}_{\mu(r)}, \hat{s}_{\eta(r)}, \dot{s}_{v(r)}\right\rangle=\left\langle\left(\dot{s}_{\mu(r)}, 0\right),\left(\dot{s}_{\eta(r)}, 0\right),\left(\dot{s}_{v(r)}, 0\right)\right\rangle .
$$

Definition 12. Let $\widetilde{\Re}=\left\langle\left(\hat{s}_{\mu}, \ddot{a}\right),\left(\hat{s}_{\eta}, \ddot{e}\right),\left(\hat{s}_{v}, \ddot{u}\right)\right\rangle$, a 2-tuple spherical fuzzy linguistic number (2TSFLN). Then, the score function of 2 TSFLN are the following;

$$
S c(\widetilde{\Re})=\Xi \sqrt{\left(\frac{\mathcal{\kappa}^{2}+\Xi^{-1}\left(\hat{s}_{\mu^{2}}, \ddot{a}\right)-\Xi^{-1}\left(\hat{s}_{\eta^{2}}, \ddot{e}\right)-\Xi^{-1}\left(\hat{s}_{v^{2}}, \ddot{u}\right)}{(3)^{2}}\right)}, \Xi^{-1}(\hat{s} c(\widetilde{\Re})) \in[1, \kappa]
$$

Definition 13. Let $\widetilde{\Re}=\left\langle\left(\hat{s}_{\mu}, \ddot{a}\right),\left(\hat{s}_{\eta}, \ddot{e}\right),\left(\hat{s}_{v}, \ddot{u}\right)\right\rangle$, a 2-tuple spherical fuzzy linguistic number (2TSFLN). Then, the accuracy function of 2TSFLN are the following;"

$$
H c(\widetilde{\Re})=\Xi \sqrt{\left(\frac{\Xi^{-1}\left(\hat{s}_{\mu^{2}}, \ddot{a}\right)+\Xi^{-1}\left(\hat{s}_{\eta^{2}}, \ddot{e}\right)+\Xi^{-1}\left(\hat{s}_{v^{2}}, \ddot{u}\right)}{(3)^{2}}\right)}, \Xi^{-1}(H c(\widetilde{\Re})) \in[1, \kappa]
$$

Definition 14. Let $\widetilde{\Re}_{1}=\left\langle\left(\hat{s}_{\mu_{1}}, \ddot{a}_{1}\right),\left(\hat{s}_{\eta_{1}}, \ddot{e}_{1}\right),\left(\hat{s}_{v_{1}}, \ddot{u}_{1}\right)\right\rangle$ and Then, if

$\widetilde{\Re}_{2}=\left\langle\left(\hat{s}_{\mu_{2}}, \ddot{a}_{2}\right),\left(\hat{s}_{\eta_{2}}, \ddot{e}_{2}\right),\left(\hat{s}_{v_{2}}, \ddot{u}_{2}\right)\right\rangle$ are the two 2-tuple spherical fuzzy linguistic number (2TSFLN).

(1) $S c\left(\widetilde{\Re}_{1}\right)>S c\left(\widetilde{\Re}_{2}\right)$, then $\widetilde{\Re}_{1}$ is grater than $\widetilde{\Re}_{2}$, if

(2) $S c\left(\widetilde{\Re}_{1}\right)=S c\left(\widetilde{\Re}_{2}\right)$, then

(3) If $H c\left(\widetilde{\Re}_{1}\right)>\operatorname{Hc}\left(\widetilde{\Re}_{2}\right)$, then $\widetilde{\Re}_{1}$ is grater than $\widetilde{\Re}_{2}$, if

(4) $H c\left(\widetilde{\Re}_{1}\right)=H c\left(\widetilde{\Re}_{2}\right)$, then $\widetilde{\Re}_{1}$ and $\widetilde{\Re}_{2}$ have the same information. 
Definition 15. Let $\widetilde{\Re}_{1}=\left\langle\left(\hat{s}_{\mu_{1}}, \ddot{a}_{1}\right),\left(\hat{s}_{\eta_{1}}, \ddot{e}_{1}\right),\left(\hat{s}_{v_{1}}, \ddot{u}_{1}\right)\right\rangle$ and $\widetilde{\Re}_{2}=\left\langle\left(\hat{s}_{\mu_{2}}, \ddot{a}_{2}\right),\left(\hat{s}_{\eta_{2}}, \ddot{e}_{2}\right),\left(\hat{s}_{v_{2}}, \ddot{u}_{2}\right)\right\rangle$ are the two 2-tuple spherical fuzzy linguistic number (2TSFLN). Then,

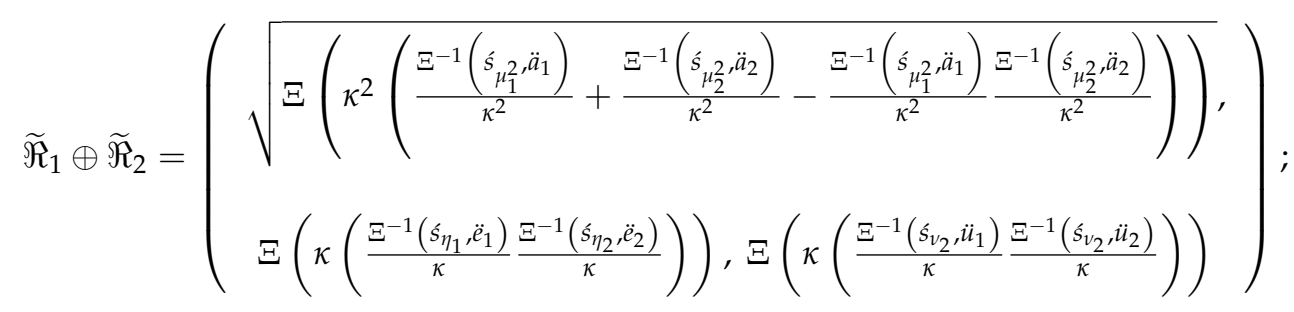

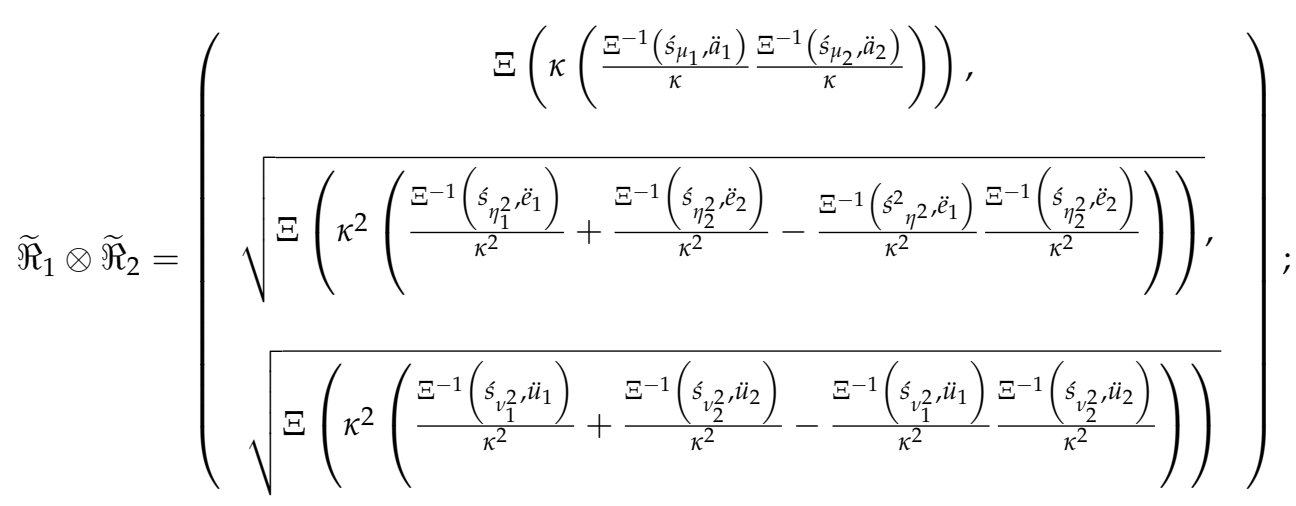

$$
\begin{aligned}
& \lambda \widetilde{\Re}_{1}=\left(\begin{array}{c}
\sqrt{\Xi\left(\kappa^{2}\left(1-\left(1-\frac{\Xi^{-1}\left(\hat{s}_{\left.\mu_{1}^{2}, \ddot{a}_{1}\right)}\right)}{\kappa^{2}}\right)\right)\right.}, \Xi\left(\kappa\left(\frac{\Xi^{-1}\left(\hat{s}_{\eta_{1}}, \ddot{e}_{1}\right)}{\kappa}\right)^{\lambda}\right), \\
\Xi\left(\kappa\left(\frac{\Xi^{-1}\left(\hat{s}_{v_{1}}, \ddot{u}_{1}\right)}{\kappa}\right)^{\lambda}\right)
\end{array} ;\right. \\
& \left(\widetilde{\Re}_{1}\right)^{\lambda}=\frac{\Xi\left(\kappa\left(\frac{\Xi^{-1}\left(\hat{s}_{\mu_{1}}, \ddot{a}_{1}\right)}{\kappa}\right)^{\lambda}\right), \sqrt{\Xi\left(\kappa^{2}\left(1-\left(1-\frac{\Xi^{-1}\left(\hat{s}_{\eta_{1}^{2}, \ddot{e}_{1}}\right)}{\kappa^{2}}\right)^{\lambda}\right)\right.}}{\sqrt{\Xi\left(\kappa^{2}\left(1-\left(1-\frac{\Xi^{-1}\left(s_{\left.v_{1}^{2}, u_{1}\right)}\right)}{\kappa^{2}}\right)\right)\right.}} .
\end{aligned}
$$

\section{2-Tuple Spherical Fuzzy Linguistic Averaging Aggregation Operators}

In this section, we use the information of 2-tuple spherical fuzzy linguistic numbers, and develop some arithmetic aggregation operators.

Definition 16. Let $\widetilde{\Re}=\left\langle\left(\hat{s}_{\mu}, \ddot{a}\right),\left(\hat{s}_{\eta}, \ddot{e}\right),\left(\hat{s}_{v}, \ddot{u}\right)\right\rangle(j=1, \ldots, n)$ be the set of 2-tuple spherical fuzzy linguistic numbers. Then, the 2-tuple spherical fuzzy linguistic weighted average (2TSFLWA) operator is a function of $\Omega^{n} \rightarrow \Omega$ such that

$$
2 \operatorname{TSFLWA} A_{\Theta}\left(\widetilde{\Re}_{1}, \ldots, \widetilde{\Re}_{n}\right)=\bigoplus_{j=1}^{n}\left(\Theta_{,} \widetilde{\Re}_{\jmath}\right),
$$

where $\Theta=\left(\Theta_{1}, \ldots, \Theta_{n}\right)^{T}$ is the weighting vector of $\widetilde{\Re}_{j}$, such that $\Theta_{j}>0, \sum_{j=1}^{n} \Theta_{j}=1$. 
Theorem 1. The aggregated value by using the 2TSFLWA operator is also a 2-tuple spherical fuzzy linguistic numbers, such that

$$
\begin{aligned}
& 2 \operatorname{TSFLWA} A_{\Theta}\left(\widetilde{\Re}_{1}, \ldots, \widetilde{\Re}_{n}\right)=\bigoplus_{j=1}^{n}\left(\Theta, \widetilde{\Re}_{j}\right) \\
& =\left(\begin{array}{c}
\sqrt{\Xi\left(\kappa ^ { 2 } \left(1-\prod_{j=1}^{n}\left(1-\frac{\left.\Xi^{-1}\left(\hat{s}_{\left.\mu_{j}^{2}, a_{j}\right)}\right)^{2}\right)}{\kappa^{2}}\right)\right.\right.}, \\
\Xi\left(\kappa \prod_{j=1}^{n}\left(\frac{\Xi^{-1}\left(s_{\eta_{j}}, \ddot{e}_{j}\right)}{\kappa}\right)^{\Theta_{j}}\right), \Xi\left(\kappa \prod_{j=1}^{n}\left(\frac{\Xi^{-1}\left(\hat{s}_{v_{j}}, \ddot{u}_{j}\right)}{\kappa}\right)^{\Theta_{j}}\right)
\end{array}\right)
\end{aligned}
$$

where $\Theta=\left(\Theta_{1}, \ldots, \Theta_{n}\right)^{T}$ is the weights of $\widetilde{\Re}_{j}$, such that $\Theta_{j}>0, \sum_{j=1}^{n} \Theta_{j}=1$.

Proof. We use the mathematical induction principle, to prove Equation (21).

(1) When $n=2$, we get

$$
\begin{aligned}
& \Theta_{1} \widetilde{\Re}_{1}=\left(\begin{array}{c}
\left.\sqrt{\Xi\left(\kappa^{2}\left(1-\left(1-\frac{\Xi^{-1}\left(\hat{s}_{\mu_{1}^{2}}, \ddot{a}_{1}\right)}{\kappa^{2}}\right)^{\Theta_{1}}\right)\right.}\right) \\
\Xi\left(\kappa\left(\frac{\Xi^{-1}\left(\hat{s}_{\eta_{1}}, \ddot{e}_{1}\right)}{\kappa}\right)^{\Theta_{1}}\right), \Xi\left(\kappa\left(\frac{\Xi^{-1}\left(\dot{s}_{v_{1}}, \ddot{u}_{1}\right)}{\kappa}\right)^{\Theta_{1}}\right)
\end{array}\right) .
\end{aligned}
$$

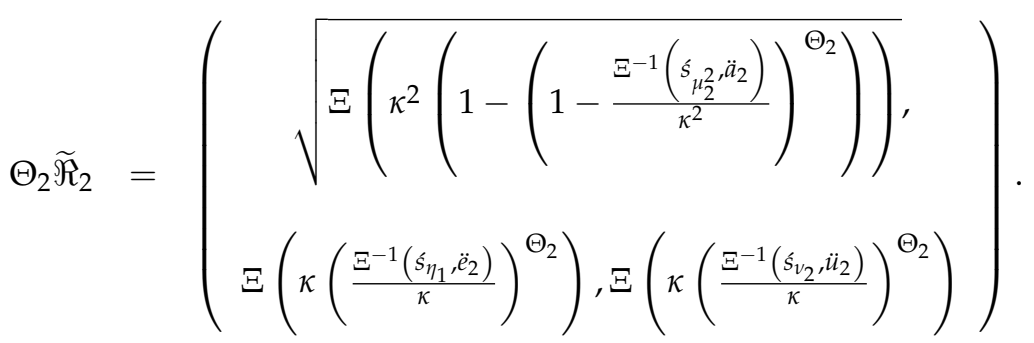

Then,

$$
2 \operatorname{TSFLWA} A_{\Theta}\left(\widetilde{\Re}_{1}, \widetilde{\Re}_{2}\right)=\left(\Theta_{1} \widetilde{\Re}_{1} \oplus \Theta_{2} \widetilde{\Re}_{2}\right)
$$

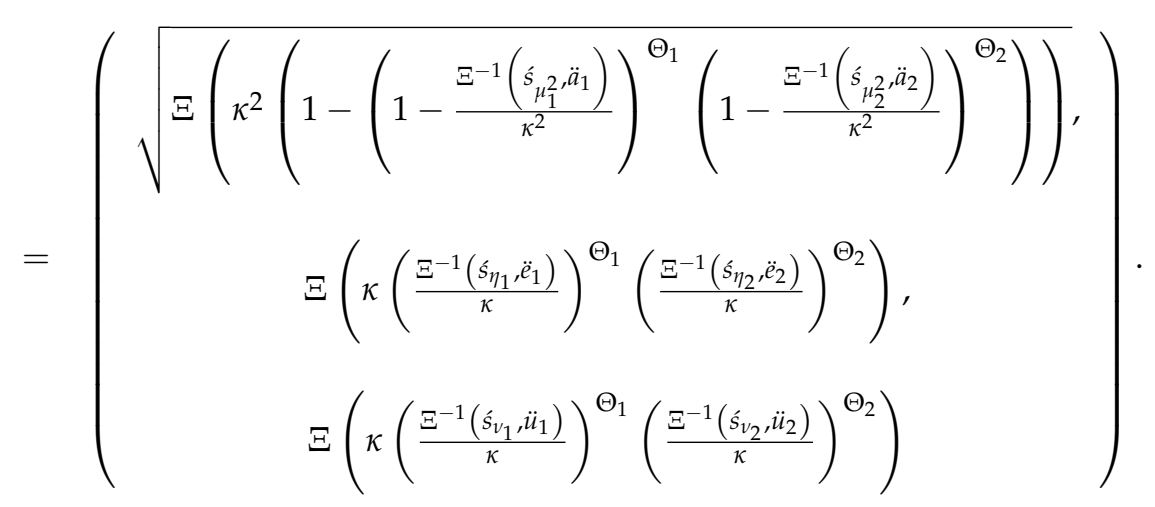


(2) Let Equation (21) be true for $n=k$, that is;

$$
\begin{aligned}
& 2 \operatorname{TSFLWA} A_{\Theta}\left(\widetilde{\Re}_{1}, \ldots, \widetilde{\Re}_{\kappa}\right)=\bigoplus_{j=1}^{k}\left(\Theta_{,} \widetilde{\Re}_{j}\right)
\end{aligned}
$$

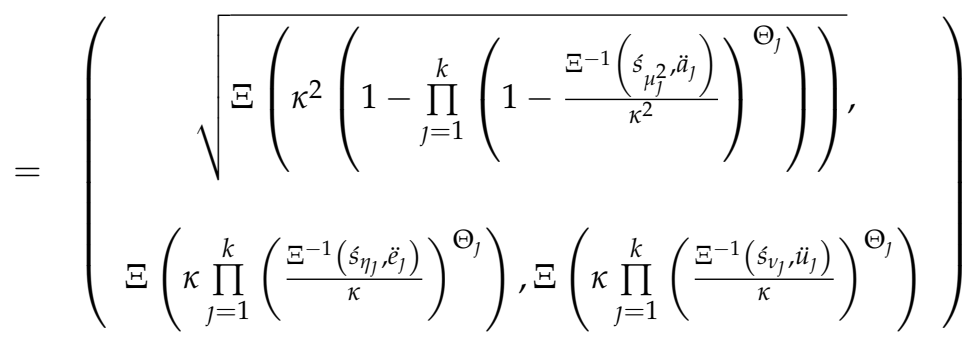

and we prove Equation (21), for $n=k+1$, then

$$
\begin{aligned}
& 2 \operatorname{TSFLWA} A_{\Theta}\left(\widetilde{\Re}_{1}, \ldots, \widetilde{\Re}_{k+1}\right)=\Theta_{1} \widetilde{\Re}_{1} \oplus \ldots \oplus \Theta_{k} \widetilde{\Re}_{k} \oplus \Theta_{k+1} \widetilde{\Re}_{k+1}
\end{aligned}
$$

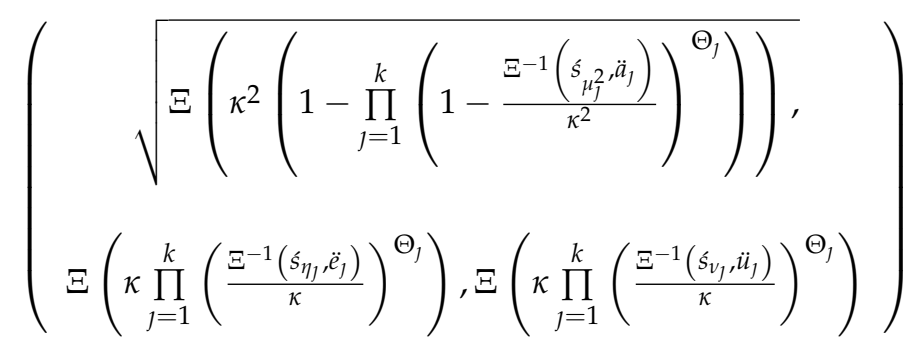

$$
\begin{aligned}
& =\left(\begin{array}{c}
\underbrace{\Xi\left(\kappa^{2}\left(1-\left(1-\frac{\Xi^{-1}\left(\hat{s}_{\mu_{\kappa+1}^{2}}^{2}, \ddot{n}_{\kappa+1}\right)}{\kappa^{2}}\right)^{\Theta_{k+1}}\right)\right)}, \\
\Xi\left(\kappa\left(\frac{\Xi^{-1}\left(\hat{s}_{\eta_{\kappa+1}}, \ddot{e}_{\kappa+1}\right)}{\kappa}\right)^{\Theta_{k+1}}\right), \Xi\left(\kappa\left(\frac{\Xi^{-1}\left(\hat{s}_{\kappa+1}, \ddot{u}_{\kappa+1}\right)}{\kappa}\right)^{\Theta_{k+1}}\right)
\end{array}\right)
\end{aligned}
$$

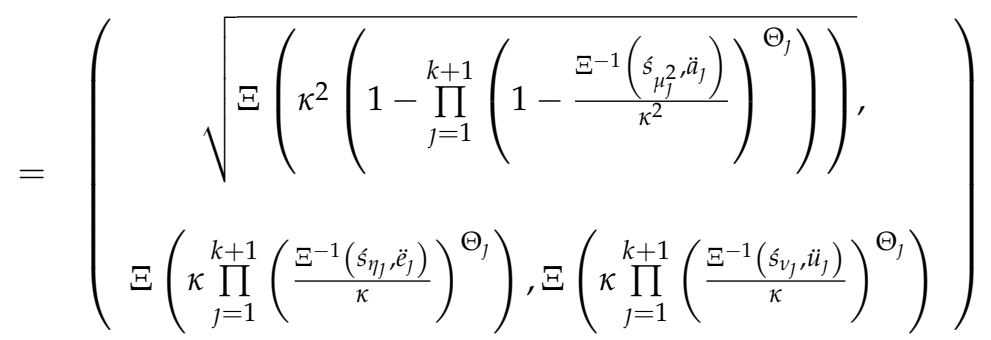

which show that the aggregated value is also a 2TSFLN. Hence Equation (21), is holds for all $n$.

Property 1 (Idempotency). If $\widetilde{\Re}_{\jmath}=\widetilde{\Re}$ for all $\mathrm{j}$, then

$$
2 \operatorname{TSFLWA} A_{\Theta}\left(\widetilde{\Re}_{1}, \ldots, \widetilde{\Re}_{n}\right)=\widetilde{\Re} .
$$

Property 2 (Boundedness). Let $\widetilde{\Re}_{j}(j=1, \ldots, n)$ be a set of 2 TSFLNs, and $\widetilde{\Re}^{+}=\max _{j} \widetilde{\Re}_{j}, \widetilde{\Re}^{-}=\min _{j} \widetilde{\Re}_{j}$, then

$$
\widetilde{\Re}^{-} \leq 2 \operatorname{TSFLWA} A_{\Theta}\left(\widetilde{\Re}_{1}, \ldots, \widetilde{\Re}_{n}\right) \leq \widetilde{\Re}^{+} .
$$


Property 3 (Monotonicity). Let $\widetilde{\Re}_{j}(j=1, \ldots, n)$ and $\widetilde{\Re}_{j}^{\prime}(j=1, \ldots, n)$ be a collection of 2 TSFLNs, if $\widetilde{\Re}_{j} \leq$ $\widetilde{\Re}_{j}^{\prime}, \forall j$, then

$$
2 \operatorname{TSFLWA} A_{\Theta}\left(\widetilde{\Re}_{1}, \ldots, \widetilde{\Re}_{n}\right) \leq 2 \operatorname{TSFLWA} A_{\Theta}\left(\widetilde{\Re}_{1}^{\prime}, \ldots, \widetilde{\Re}_{n}^{\prime}\right) .
$$

Definition 17. Let $\widetilde{\Re}=\left\langle\left(\hat{s}_{\mu}, \ddot{a}\right),\left(\hat{s}_{\eta}, \ddot{e}\right),\left(\hat{s}_{v}, \ddot{u}\right)\right\rangle(\jmath=1, \ldots, n)$ be the set of 2-tuple spherical fuzzy linguistic numbers. Then, the 2-tuple spherical fuzzy linguistic ordered weighted averaging (2TSFLOWA) operator is a function of $\Omega^{n} \rightarrow \Omega$, that has $\Theta=\left(\Theta_{1}, \ldots, \Theta_{n}\right)^{T}$ be the associated weights, and $\Theta_{j}>0, \sum_{j=1}^{n} \Theta_{j}=1$. Then,

$$
\begin{aligned}
& \operatorname{2TSFLOWA} A_{\Theta}\left(\widetilde{\Re}_{1}, \ldots, \widetilde{\Re}_{n}\right)=\bigoplus_{j=1}^{n}\left(\Theta, \widetilde{\Re}_{\sigma(\jmath)}\right)
\end{aligned}
$$

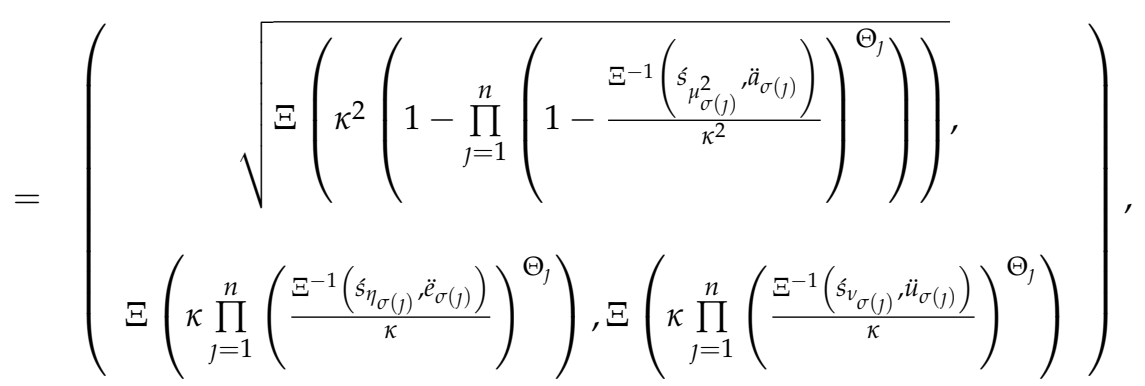

where the permutation of $(1, \ldots, n)$ is $\left(\sigma(1), \ldots, \sigma(n)\right.$, and defined as $\widetilde{\Re}_{\sigma(\jmath-1)} \geq \widetilde{\Re}_{\sigma(j)} \forall j=2, \ldots, n$.

The 2TSFLOWA operator has the following properties.

Property 4 (Idempotency). If $\widetilde{\Re}_{\jmath}=\widetilde{\Re}$ for all $\mathrm{j}$, then

$$
2 \operatorname{TSFLOWA} A_{\Theta}\left(\widetilde{\Re}_{1}, \ldots, \widetilde{\Re}_{n}\right)=\widetilde{\Re} \text {. }
$$

Property 5 (Boundedness). Let $\widetilde{\Re}_{j}(j=1, \ldots, n)$ be the collection of 2 TSFLNs, and $\widetilde{\Re}^{+}=\max _{j} \widetilde{\Re}_{j}, \widetilde{\Re}^{-}=$ $\min _{j} \widetilde{\Re}$. Then,

$$
\widetilde{\Re}^{-} \leq 2 \operatorname{TSFLOWA} A_{\Theta}\left(\widetilde{\Re}_{1}, \ldots, \widetilde{\Re}_{n}\right) \leq \widetilde{\Re}^{+} .
$$

Property 6 (Monotonicity). Let $\widetilde{\Re}_{j}(j=1, \ldots, n)$ and $\widetilde{\Re}_{j}^{\prime}(j=1, \ldots, n)$ be the collection of 2TSFLNs, if $\widetilde{\Re}_{j} \leq \widetilde{\Re}_{j}^{\prime}, \forall j$. Then,

$$
2 \operatorname{TSFLOWA} A_{\Theta}\left(\widetilde{\Re}_{1}, \ldots, \widetilde{\Re}_{n}\right) \leq 2 \operatorname{TSFLOW} A_{\Theta}\left(\widetilde{\Re}_{1}^{\prime}, \ldots, \widetilde{\Re}_{n}^{\prime}\right)
$$

Definition 18. Let $\widetilde{\Re}=\left\langle\left(\hat{s}_{\mu}, \ddot{a}\right),\left(\hat{s}_{\eta}, \ddot{e}\right),\left(\hat{s}_{v}, \ddot{u}\right)\right\rangle(\jmath=1, \ldots, n)$ be the set of 2-tuple spherical fuzzy linguistic numbers. Then, the 2-tuple spherical fuzzy linguistic hybrid average (2TSFLHA) operator is a function of $\Omega^{n} \rightarrow \Omega$, such that

$$
\begin{aligned}
& 2 \operatorname{TSFLHA} A_{\Theta}\left(\widetilde{\Re}_{1}, \ldots, \widetilde{\Re}_{n}\right)=\bigoplus_{j=1}^{n}\left(\Theta, \widetilde{\Re}_{\sigma(j)}\right)
\end{aligned}
$$

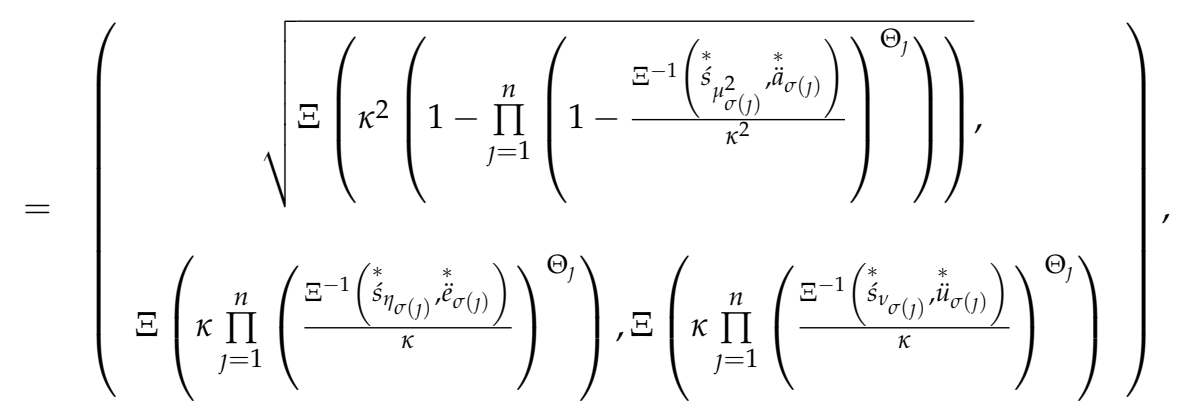


where $\Theta=\left(\Theta_{1}, \ldots, \Theta_{n}\right)^{T}$ is the associated weights, such that $\Theta_{j}>0, \sum_{j=1}^{n} \Theta_{j}=1$, and $\widetilde{\widetilde{R}}_{\sigma(\jmath)}$ is the $j^{\text {th }}$

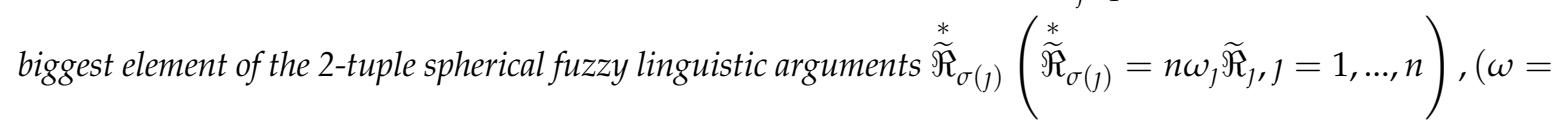
$\left.\omega_{1}, \ldots, \omega_{n}\right)$ is the weight vector of 2-tuple spherical fuzzy linguistic arguments $\widetilde{\Re}_{j}$, with $\omega_{j}>0, \sum_{j=1}^{n} \omega_{j}=1$, and $n$ is the balancing coefficient.

\section{2-Tuple Spherical Fuzzy Linguistic Geometric Aggregation Operators}

In this section, we use the information of 2-tuple spherical fuzzy linguistic numbers, and develop some geometric aggregation operators.

Definition 19. Let $\widetilde{\Re}=\left\langle\left(\hat{s}_{\mu}, \ddot{a}\right),\left(\hat{s}_{\eta}, \ddot{e}\right),\left(\hat{s}_{v}, \ddot{u}\right)\right\rangle(\jmath=1, \ldots, n)$ be the set of 2-tuple spherical fuzzy linguistic numbers. Then, the 2-tuple spherical fuzzy linguistic weighted geometric (2TSFLWG) operator is a function of $\Omega^{n} \rightarrow \Omega$, such that

$$
2 \operatorname{TSFLWG} G_{\Theta}\left(\widetilde{\Re}_{1}, \ldots, \widetilde{\Re}_{n}\right)=\bigotimes_{j=1}^{n}\left(\widetilde{\Re}_{\jmath}\right)^{\Theta_{j}},
$$

where $\Theta=\left(\Theta_{1}, \ldots, \Theta_{n}\right)^{T}$ is the weighting vector of $\widetilde{\Re}_{j}$, such that $\Theta_{j}>0, \sum_{j=1}^{n} \Theta_{j}=1$.

Theorem 2. The aggregated value by using 2 TSFLWG operator is also a 2-tuple spherical fuzzy linguistic numbers, such that

$$
\begin{aligned}
& 2 \operatorname{TSFLWG} G_{\Theta}\left(\widetilde{\Re}_{1}, \ldots, \widetilde{\Re}_{n}\right)=\bigotimes_{j=1}^{n}\left(\widetilde{\Re}_{\jmath}\right)^{\Theta_{\jmath}}
\end{aligned}
$$

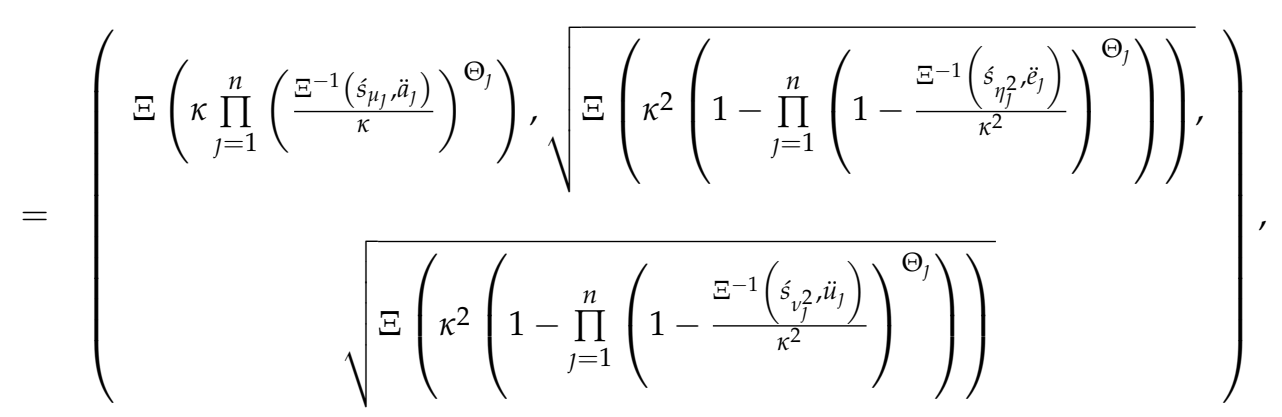

where $\Theta=\left(\Theta_{1}, \ldots, \Theta_{n}\right)^{T}$ is the weighs of $\widetilde{\Re}_{j}$, such that $\Theta_{j}>0, \sum_{j=1}^{n} \Theta_{j}=1$.

Proof. We use the mathematical induction principle, to prove Equation (31). 
(1) When $n=2$, we get

$$
\begin{aligned}
& \left(\widetilde{\Re}_{1}\right)^{\Theta_{1}}=\underbrace{\left.\Xi\left(\kappa\left(\frac{\Xi^{-1}\left(\hat{s}_{\mu_{1}}, \ddot{a}_{1}\right)}{\kappa}\right)^{\Theta_{1}}\right), \sqrt{\Xi\left(\kappa^{2}\left(1-\left(1-\frac{\Xi^{-1}\left(\hat{s}_{\eta_{1}^{2}, \ddot{e}_{1}}\right)}{\kappa^{2}}\right)^{\Theta_{1}}\right)\right.}\right)} . \\
& \left(\widetilde{\Re}_{2}\right)^{\Theta_{2}}=\underbrace{\Xi\left(\kappa\left(\frac{\Xi^{-1}\left(\dot{s}_{\mu_{1}}, \ddot{a}_{2}\right)}{\kappa}\right)^{\Theta_{2}}\right), \sqrt{\Xi\left(\kappa^{2}\left(1-\left(1-\frac{\Xi^{-1}\left(s_{\left.\eta_{2}^{2}, \ddot{e}_{2}\right)}\right)^{2}}{\Xi\left(\kappa ^ { 2 } \left(1-\left(1-\frac{\Xi^{-1}\left(s_{v_{2}, \ddot{u}_{2}}\right)}{\kappa^{2}}\right)\right.\right.}\right)^{\Theta_{2}}\right)\right)}} .
\end{aligned}
$$

Then,

$$
\begin{aligned}
& \operatorname{TTSFLWG}\left(\widetilde{\Re}_{1}, \widetilde{\Re}_{2}\right)=\left(\widetilde{\Re}_{1}\right)^{\Theta_{1}} \otimes\left(\widetilde{\Re}_{2}\right)^{\Theta_{2}}
\end{aligned}
$$

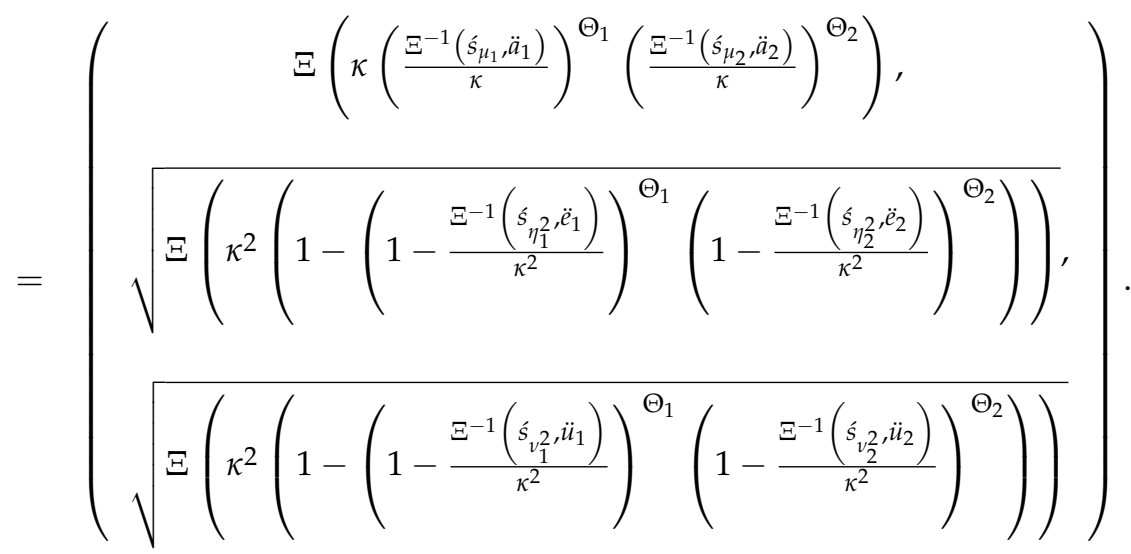

(2) Assume that Equation (31), is true for $n=k$, that is;

$$
\begin{aligned}
& 2 \operatorname{TSFLWG} G_{\Theta}\left(\widetilde{\Re}_{1}, \ldots, \widetilde{\Re}_{\kappa}\right)=\bigoplus_{j=1}^{k}\left(\widetilde{\Re}_{\jmath}\right)^{\Theta_{j}}
\end{aligned}
$$

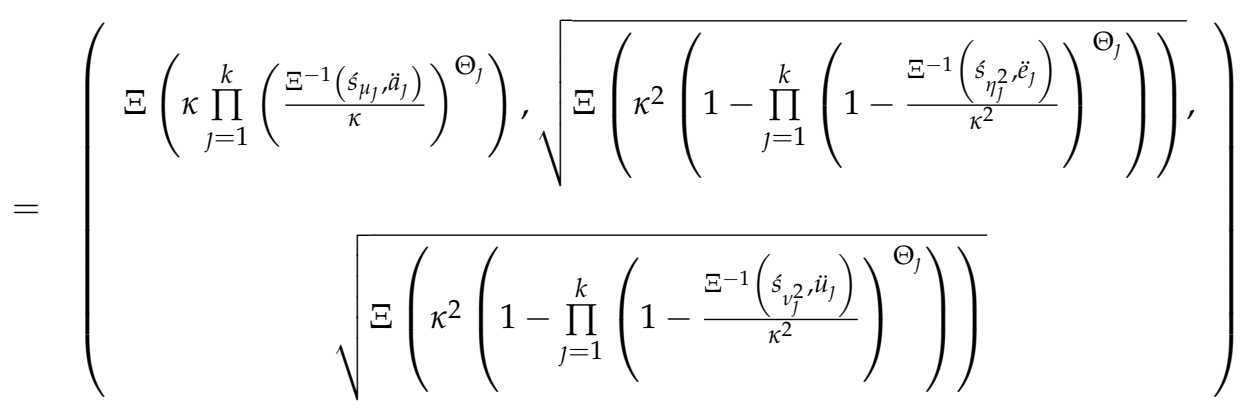


and we prove Equation (31), for $n=k+1$, then

$$
\begin{aligned}
& 2 T S F L W G\left(\widetilde{\Re}_{1}, \ldots, \widetilde{\Re}_{k+1}\right)=\left(\widetilde{\Re}_{1}\right)^{\Theta_{1}} \otimes \ldots \otimes\left(\widetilde{\Re}_{k}\right)^{\Theta_{k}} \otimes\left(\widetilde{\Re}_{k+1}\right)^{\Theta_{k+1}}
\end{aligned}
$$

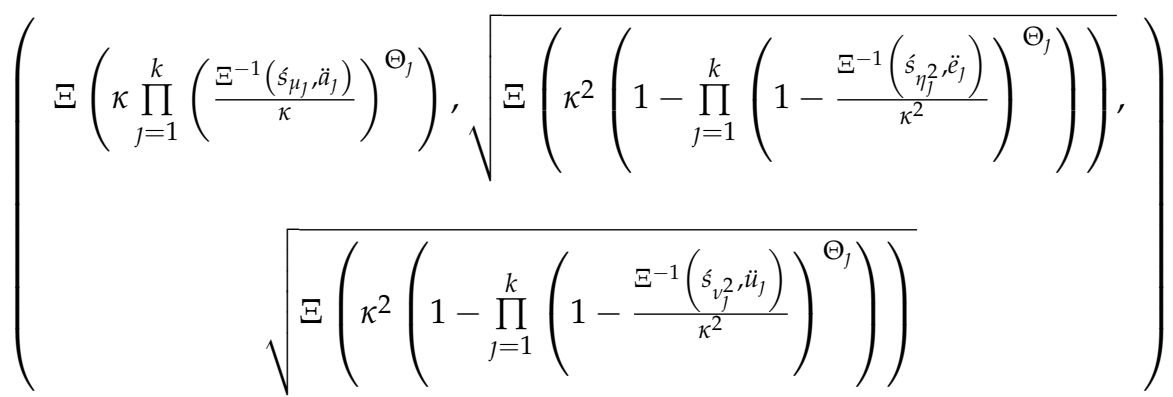

$$
\begin{aligned}
& = \\
& \otimes\left(\sqrt{\Xi\left(\kappa\left(\frac{\Xi^{-1}\left(\hat{s}_{\mu_{k+1}}, \ddot{n}_{\kappa+1}\right)}{\kappa}\right){ }^{\Theta_{k+1}}\right), \sqrt{\Xi\left(\kappa^{2}\left(1-\left(1-\frac{\Xi^{-1}\left(\hat{s}_{\eta_{k+1}^{2}}, \ddot{e}_{\kappa+1}\right)}{\kappa^{2}}\right)^{\Theta_{k+1}}\right)\right.},}\right)
\end{aligned}
$$

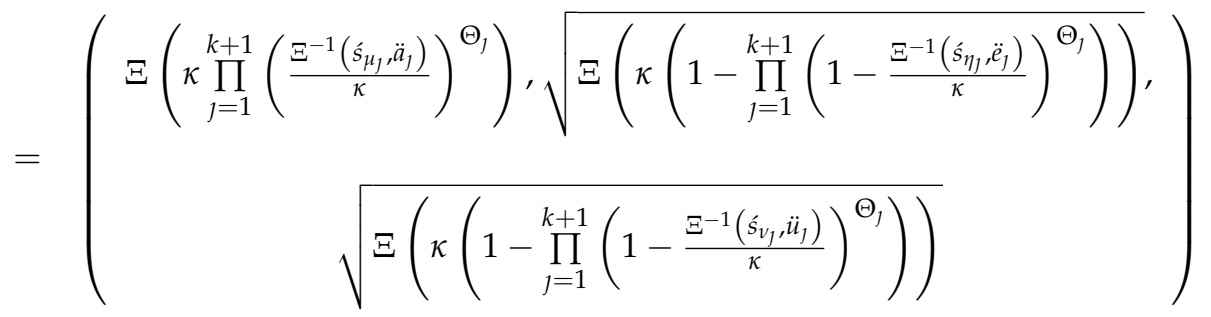

which show that the aggregated value is also a 2TSFLN. Hence Equation (31) holds for all $n$.

The 2TSFLWG operator satisfies the following properties.

Property 7 (Idempotency). If $\widetilde{\Re}_{\jmath}=\widetilde{\Re}$ for all $\mathrm{j}$, then

$$
2 \operatorname{TSFLWG}\left(\widetilde{\Re}_{1}, \ldots, \widetilde{\Re}_{n}\right)=\widetilde{\Re} .
$$

Property 8 (Boundedness). Let $\widetilde{\Re}_{j}(j=1, \ldots, n)$ be a set of 2 TSFLNs, and $\widetilde{\Re}^{+}=\max _{j} \widetilde{\Re}_{j}, \widetilde{\Re}^{-}=\min _{j} \widetilde{\Re}_{j}$, then

$$
\widetilde{\Re}^{-} \leq 2 \operatorname{TSFLWG}\left(\widetilde{\Re}_{1}, \ldots, \widetilde{\Re}_{n}\right) \leq \widetilde{\Re}^{+} .
$$

Property 9 (Monotonicity). Let $\widetilde{\Re}_{j}(j=1, \ldots, n)$ and $\widetilde{\Re}_{j}^{\prime}(j=1, \ldots, n)$ be a collection of 2 TSFLNs, if $\widetilde{\Re}_{j} \leq$ $\widetilde{\Re}_{j}^{\prime}, \forall j$, then

$$
2 \operatorname{TSFLWG}\left(\widetilde{\Re}_{1}, \ldots, \widetilde{\Re}_{n}\right) \leq 2 \operatorname{TSFLWG}\left(\widetilde{\Re}_{1}^{\prime}, \ldots, \widetilde{\Re}_{n}^{\prime}\right) .
$$


Definition 20. Let $\widetilde{\Re}=\left\langle\left(\hat{s}_{\mu}, \ddot{a}\right),\left(\hat{s}_{\eta}, \ddot{e}\right),\left(\hat{s}_{v}, \ddot{u}\right)\right\rangle(\jmath=1, \ldots, n)$ be the set of 2-tuple spherical fuzzy linguistic numbers. Then, the 2-tuple spherical fuzzy linguistic ordered weighted geometric (2TSFLOWG) operator is a function of $\Omega^{n} \rightarrow \Omega$, that has $\Theta=\left(\Theta_{1}, \ldots, \Theta_{n}\right)^{T}$ be the associated weights, and $\Theta_{j}>0, \sum_{j=1}^{n} \Theta_{j}=1$. Then,

$$
\begin{aligned}
& 2 \operatorname{TSFLOWG}\left(\widetilde{\Re}_{1}, \ldots, \widetilde{\Re}_{n}\right)=\bigotimes_{j=1}^{n}\left(\widetilde{\Re}_{\sigma(\jmath)}\right)^{\Theta_{j}}
\end{aligned}
$$

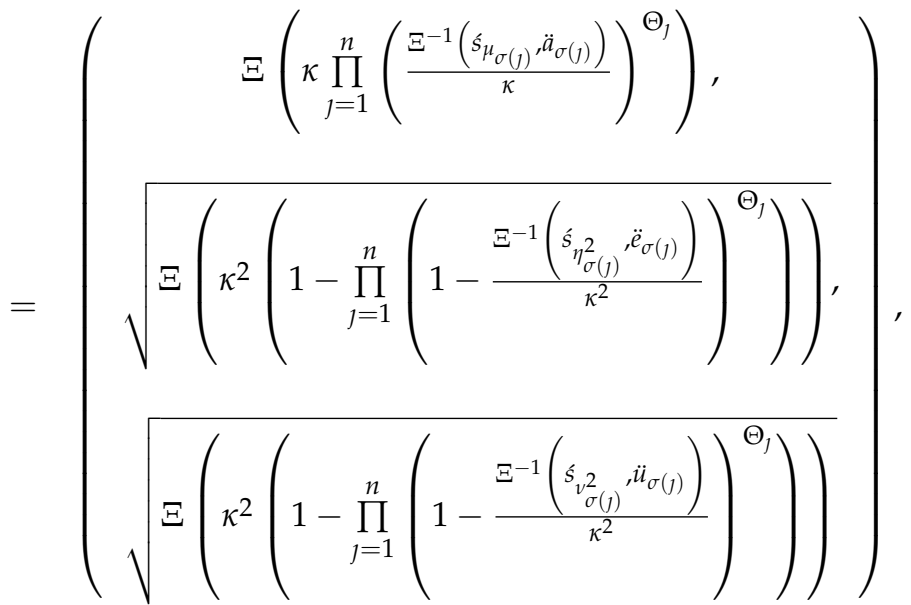

where $\left(\sigma(1), \ldots, \sigma(n)\right.$ is a permutation of $(1, \ldots, n)$, and $\widetilde{\Re}_{\sigma(\jmath-1)} \geq \widetilde{\Re}_{\sigma(\jmath)} \forall j=2, \ldots, n$.

The 2TSFLOWG operator has the following properties.

Property 10 (Idempotency). If $\widetilde{\Re}_{j}=\widetilde{\Re}$ for all j, then

$$
2 \operatorname{TSFLOWG} G_{\Theta}\left(\widetilde{\Re}_{1}, \ldots, \widetilde{\Re}_{n}\right)=\widetilde{\Re} .
$$

Property 11 (Boundedness). Let $\widetilde{\Re}_{j}(j=1, \ldots, n)$ be the collection of 2 TSFLNs, and $\widetilde{\Re}^{+}=\max _{j} \widetilde{\Re}_{j}, \widetilde{\Re}^{-}=$ $\min _{j} \widetilde{\Re}_{j}$. Then,

$$
\widetilde{\Re}^{-} \leq 2 \operatorname{TSFLOWG}\left(\widetilde{\Re}_{1}, \ldots, \widetilde{\Re}_{n}\right) \leq \widetilde{\Re}^{+} .
$$

Property 12 (Monotonicity). Let $\widetilde{\Re}_{j}(j=1, \ldots, n)$ and $\widetilde{\Re}_{j}^{\prime}(j=1, \ldots, n)$ be the collection of 2TSFLNs, if $\widetilde{\Re}_{j} \leq \widetilde{\Re}_{j}^{\prime}, \forall j$. Then,

$$
2 \operatorname{TSFLOWG} G_{\Theta}\left(\widetilde{\Re}_{1}, \ldots, \widetilde{\Re}_{n}\right) \leq 2 \operatorname{TSFLOWG} G_{\Theta}\left(\widetilde{\Re}_{1}^{\prime}, \ldots, \widetilde{\Re}_{n}^{\prime}\right) .
$$


Definition 21. Let $\widetilde{\Re}=\left\langle\left(\hat{s}_{\mu}, \ddot{a}\right),\left(\hat{s}_{\eta}, \ddot{e}\right),\left(\hat{s}_{v}, \ddot{u}\right)\right\rangle(\jmath=1, \ldots, n)$ be the set of 2-tuple spherical fuzzy linguistic numbers. Then, the 2-tuple spherical fuzzy linguistic hybrid geometric (2TSFLHG) operator is a function of $\Omega^{n} \rightarrow \Omega$, such that

$$
\begin{aligned}
& 2 \operatorname{TSFLHG}\left(\widetilde{\Re}_{\Theta}, \ldots, \widetilde{\Re}_{n}\right)=\bigotimes_{j=1}^{n}\left(\widetilde{\widetilde{R}}_{\sigma(\jmath)}^{*}\right)^{\Theta_{j}}
\end{aligned}
$$

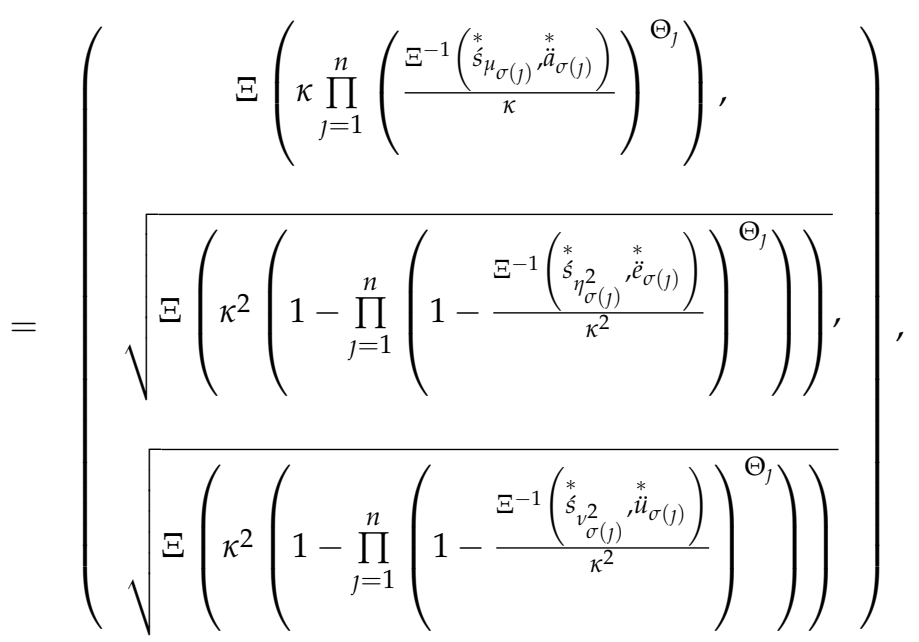

where $\Theta=\left(\Theta_{1}, \ldots, \Theta_{n}\right)^{T}$ is the associated weights, such that $\Theta_{j}>0, \sum_{j=1}^{n} \Theta_{j}=1$, and $\stackrel{*}{\widetilde{R}}_{\sigma(j)}$ is the $j^{\text {th }}$ biggest

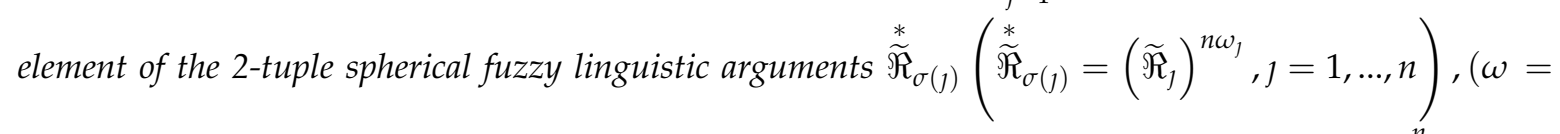
$\left.\omega_{1}, \ldots, \omega_{n}\right)$ is the weighting vector of 2-tuple spherical fuzzy linguistic arguments $\widetilde{\Re}_{j}$, with $\omega_{j}>0, \sum_{j=1}^{n} \omega_{j}=1$, and $n$ is the balancing coefficient.

\section{An Approach for MADM with 2-Tuple Spherical Fuzzy Linguistic Information}

Based on these two operators (2TSFLWA or 2TSFLWG operators) in this section, we propose a model for MADM problem, with the 2-tuple spherical fuzzy linguistic information. Suppose that the discrete set of alternatives are $\mathbb{Q}=\left(\mathbb{Q}_{1}, \ldots, \mathbb{Q}_{m}\right)$, and the attributes set are $\mathbb{N}=\left(\mathbb{N}_{1}, \ldots, \mathbb{N}_{n}\right)$, where $\Theta=\left(\Theta_{1}, \ldots, \Theta_{n}\right)^{T}$ is the weights of the attribute $\mathbb{N}_{j}$, and $\Theta_{j} \in[0,1], \sum_{j=1}^{n} \Theta_{j}=1$. Assume that $\mathbb{Z}=\left(\widetilde{r}_{l j}\right)_{m \times n}=\left\langle\left(\hat{s}_{\mu}, \ddot{a}\right),\left(\hat{s}_{\eta}, \ddot{e}\right),\left(\hat{s}_{v}, \ddot{u}\right)\right\rangle_{m \times n}$ is the 2-tuple spherical fuzzy linguistic decision matrix, where $\widetilde{r}_{l j}$, take the form of the 2-tuple spherical fuzzy linguistic numbers, and $\left(\hat{s}_{\mu}, \ddot{a}\right),\left(\hat{s}_{\eta}, \ddot{e}\right),\left(\hat{s}_{v}, \ddot{u}\right)$ denote the positive, neutral, and negative grades, respectively, that the alternative $\mathbb{Q}_{\text {l }}$ satisfies the attribute $\mathbb{N}_{\text {}}$ given by the decision makers. Where $\left(\hat{s}_{\mu}, \ddot{a}\right),\left(\hat{s}_{\eta}, \ddot{e}\right),\left(\hat{s}_{v}, \ddot{u}\right) \in \hat{S}_{,}, \ddot{a}_{\imath}, \ddot{e}_{l}, \ddot{u}_{\imath} \leq[-0.5,0.5), \imath=$ $1, \ldots, m ; \jmath=1, \ldots, n$. Now, we used the 2-tuple Sphercial fuzzy linguistic information and apply the 2TSFLWA or 2TSFLWG operator for the MADM problem. 
Step 1. To find the total preference values $\widetilde{\Re}_{\imath}(\imath=1, \ldots, m)$ of the alternative $\mathbb{Q}_{1}$, we used the given information of the matrix $\mathbb{Z}$, and the 2TSFLWA (2TSFLWG) operators.

$$
\begin{aligned}
& 2 \operatorname{TSFLWA}\left(\widetilde{\Re}_{1}, \ldots, \widetilde{\Re}_{n}\right)=\bigoplus_{j=1}^{n}\left(\Theta_{j} \widetilde{\Re}_{j}\right)
\end{aligned}
$$

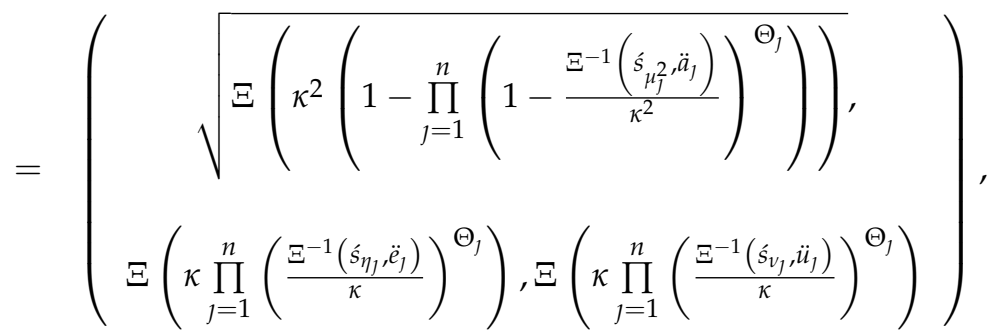

Or

$$
\begin{aligned}
& 2 T S F L W G_{\Theta}\left(\widetilde{\Re}_{1}, \ldots, \widetilde{\Re}_{n}\right)=\bigotimes_{j=1}^{n}\left(\widetilde{\Re}_{\jmath}\right)^{\Theta_{\jmath}}
\end{aligned}
$$

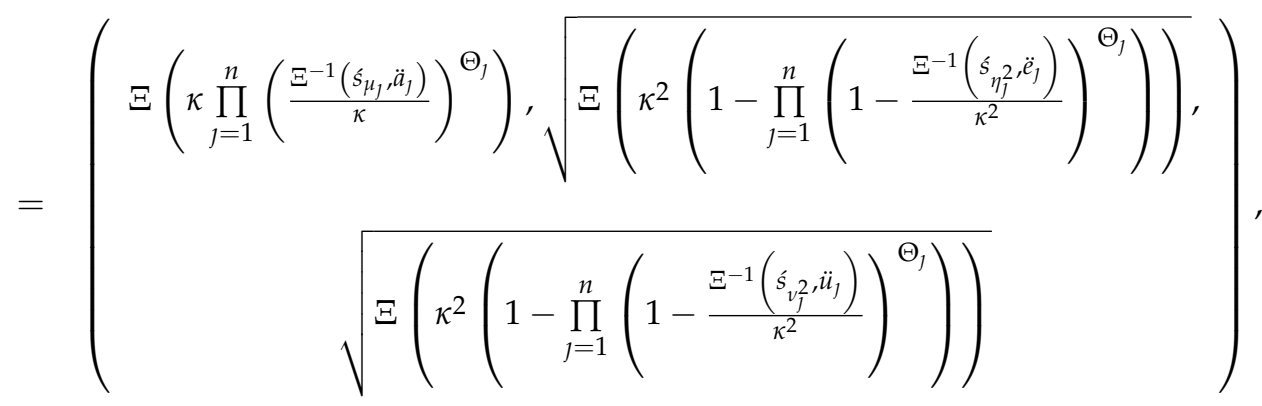

Step 2. Find the scores $S c\left(\widetilde{\Re}_{\imath}\right)(\imath=1, \ldots, m)$ values of the total 2-tuple spherical fuzzy linguistic numbers $\widetilde{\Re}_{1}$.

Step 3. According to the score value $S c\left(\widetilde{\Re}_{\imath}\right)(\imath=1, \ldots, m)$, give ranking to the alternatives $\mathbb{Q}_{l}$ and select the best one.

\section{Practical Example}

In 1962 commercial poultry was established in Pakistan. It was the largest Agro-based segment of Pakistan having investment of more than 750,000,000,000 rupees. Poultry industry of Pakistan is making large contributions in bridging the gap between supply and demand of meat protein and contributes $1.4 \%$ of national GDP. With continuous depletion of supply of red meat poultry is the cheapest available animal protein source for our masses and such is an effective check upon the spiraling animal protein price. Therefore we are already consuming less protein as per the required standard, because of the lesser supply of meat available to people. One problem of less supply of broiler chicken meat is not using good feeds for it because the farmers are not aware about feeds. In Pakistan there are three main feeds companies (alternatives) producing the feeds which are Jadeed, Islamabad and Sadiqa brothers. Unfortunately, the former does not take a good decision on better company feeds for more broiler chicken weight, giving a higher per $\mathrm{kg}$ price and generating more revenue from the flock. For the best company (alternative) selection for the feeds, we have four attributes (1) weight of the chicken, (2) price of the feeds, (3) quality of chicken, and (4) price of chicken, with the weighting vector $\Theta=(0.27,0.24,0.23,0.26)^{T}$, which is obtained by using the method of [52]. Now to select the best feeds company, we collect the data from the different forms, and write in the form of 2-tuple spherical fuzzy numbers. The given data are presented in Table 1 . Then, we apply the aggregation operators on the given data, and select the best company (alternative). 
Table 1. 2-tuple spherical fuzzy linguistic decision matrix.

\begin{tabular}{|c|c|c|c|c|}
\hline & $\mathbb{N}_{1}$ & $\mathbb{N}_{2}$ & $\mathbb{N}_{3}$ & $\mathbb{N}_{4}$ \\
\hline $\begin{array}{l}\mathbb{Q}_{1} \\
\mathbb{Q}_{2} \\
\mathbb{Q}_{3}\end{array}$ & $\begin{array}{l}\left\langle\left(s_{8}, 0\right),\left(s_{3}, 0\right)\left(s_{3}, 0\right)\right\rangle \\
\left\langle\left(s_{6}, 0\right),\left(s_{4}, 0\right)\left(s_{4}, 0\right)\right\rangle \\
\left\langle\left(s_{7}, 0\right),\left(s_{3}, 0\right)\left(s_{4}, 0\right)\right\rangle\end{array}$ & $\begin{array}{l}\left\langle\left(s_{6}, 0\right),\left(s_{4}, 0\right)\left(s_{3}, 0\right)\right\rangle \\
\left\langle\left(s_{7}, 0\right),\left(s_{3}, 0\right)\left(s_{4}, 0\right)\right\rangle \\
\left\langle\left(s_{6}, 0\right),\left(s_{4}, 0\right)\left(s_{3}, 0\right)\right\rangle\end{array}$ & $\begin{array}{l}\left\langle\left(s_{6}, 0\right),\left(s_{4}, 0\right)\left(s_{3}, 0\right)\right\rangle \\
\left\langle\left(s_{5}, 0\right),\left(s_{3}, 0\right)\left(s_{4}, 0\right)\right\rangle \\
\left\langle\left(s_{3}, 0\right),\left(s_{4}, 0\right)\left(s_{5}, 0\right)\right\rangle\end{array}$ & $\begin{array}{l}\left\langle\left(s_{6}, 0\right),\left(s_{4}, 0\right)\left(s_{5}, 0\right)\right\rangle \\
\left\langle\left(s_{3}, 0\right),\left(s_{5}, 0\right)\left(s_{6}, 0\right)\right\rangle \\
\left\langle\left(s_{4}, 0\right),\left(s_{4}, 0\right)\left(s_{4}, 0\right)\right\rangle\end{array}$ \\
\hline
\end{tabular}

To choose the most desirable company, we used the following steps (Tables 2-4);

Table 2. The aggregated values of alternative by the 2TSFLWA (2TSFLWG) operators.

\begin{tabular}{ccc}
\hline & 2TSFLWA & 2TSFLWG \\
\hline $\mathbb{Q}_{1}$ & $\left\langle\left(\hat{s}_{7},-0.19\right),\left(\hat{s}_{4},-0.30\right),\left(\hat{s}_{3}, 0.42\right)\right\rangle$ & $\left\langle\left(\hat{s}_{6}, 0.48\right),\left(\hat{s}_{4},-0.23\right),\left(\hat{s}_{4},-0.32\right)\right\rangle$ \\
$\mathbb{Q}_{2}$ & $\left\langle\left(\hat{s}_{6},-0.37\right),\left(\hat{s}_{4},-0.30\right),\left(\hat{s}_{4}, 0.44\right)\right\rangle$ & $\left\langle\left(\dot{s}_{5},-0.02\right),\left(\hat{s}_{4},-0.08\right),\left(\dot{s}_{5},-0.32\right)\right\rangle$ \\
$\mathbb{Q}_{3}$ & $\left\langle\left(\dot{s}_{6},-0.47\right),\left(\hat{s}_{4},-0.31\right),\left(\hat{s}_{4},-0.08\right)\right\rangle$ & $\left\langle\left(\hat{s}_{5},-0.20\right),\left(\hat{s}_{4},-0.23\right),\left(\dot{s}_{4}, 0.08\right)\right\rangle$ \\
\hline
\end{tabular}

Table 3. Alternative (company) score values.

\begin{tabular}{ccc}
\hline & 2TSFLWA & 2TSFLWG \\
\hline $\mathbb{Q}_{1}$ & $\left(s_{3}, 0.41\right)$ & $\left(s_{3}, 0.09\right)$ \\
$\mathbb{Q}_{2}$ & $\left(s_{3}, 0.06\right)$ & $\left(s_{3},-0.31\right)$ \\
$\mathbb{Q}_{3}$ & $\left(s_{3}, 0.08\right)$ & $\left(s_{3},-0.13\right)$ \\
\hline
\end{tabular}

Table 4. Ranking of the alternatives.

\begin{tabular}{lc}
\hline Operators & Ranking \\
\hline 2TSFLWA & $\mathbb{Q}_{1}>\mathbb{Q}_{3}>\mathbb{Q}_{2}$ \\
2TSFLWG & $\mathbb{Q}_{1}>\mathbb{Q}_{3}>\mathbb{Q}_{2}$ \\
\hline
\end{tabular}

\section{A Comparative Analysis with Linguistic Spherical Fuzzy Sets}

The concept of linguistic spherical fuzzy sets was introduced by Jin et al. [47].

First of all, we convert the data of created problem to linguistic spherical fuzzy numbers, which are shown in Table 5.

Table 5. The linguistic spherical fuzzy numbers decision matrix.

\begin{tabular}{|c|c|c|c|c|}
\hline & $\mathbb{N}_{1}$ & $\mathbb{N}_{2}$ & $\mathbb{N}_{3}$ & $\mathbb{N}_{4}$ \\
\hline $\begin{array}{l}\mathbb{Q}_{1} \\
\mathbb{Q}_{2} \\
\mathbb{Q}_{3}\end{array}$ & $\begin{array}{l}\left\langle\left(\dot{s}_{8}, \hat{s}_{3}, \dot{s}_{3}\right)\right\rangle \\
\left\langle\left(\dot{s}_{6}, \hat{s}_{4}, \dot{s}_{4}\right)\right\rangle \\
\left\langle\left(\dot{s}_{7}, \dot{s}_{3}, \dot{s}_{4}\right)\right\rangle\end{array}$ & $\begin{array}{l}\left\langle\left(\dot{s}_{6}, \dot{s}_{4}, \dot{s}_{3}\right)\right\rangle \\
\left\langle\left(\dot{s}_{7}, \hat{s}_{3}, \dot{s}_{4}\right)\right\rangle \\
\left\langle\left(\dot{s}_{6}, \hat{s}_{4}, \dot{s}_{3}\right)\right\rangle\end{array}$ & $\begin{array}{l}\left\langle\left(\dot{s}_{6}, \hat{s}_{4}, \hat{s}_{3}\right)\right\rangle \\
\left\langle\left(\dot{s}_{5}, \dot{s}_{3}, \dot{s}_{4}\right)\right\rangle \\
\left\langle\left(\dot{s}_{3}, \dot{s}_{4}, \dot{s}_{5}\right)\right\rangle\end{array}$ & $\begin{array}{l}\left\langle\left(\hat{s}_{6}, \hat{s}_{4}, \hat{s}_{5}\right)\right\rangle \\
\left\langle\left(\hat{s}_{3}, \hat{s}_{5}, \dot{s}_{6}\right)\right\rangle \\
\left\langle\left(\dot{s}_{4}, \hat{s}_{4}, \hat{s}_{4}\right)\right\rangle\end{array}$ \\
\hline
\end{tabular}

Then, we apply all the steps of Jin et al. [47] approach, and using the weighting vector of the attributes are $(0.27,0.24,0.23,0.26)^{T}$. We obtain the following ranking (Table 6);

Table 6. Ordering of the alternatives.

\begin{tabular}{cc}
\hline Operators & Ordering \\
\hline LSFWA [47] & $\mathbb{Q}_{1}>\mathbb{Q}_{3}>\mathbb{Q}_{2}$ \\
\hline LSFWG [47] & $\mathbb{Q}_{1}>\mathbb{Q}_{3}>\mathbb{Q}_{2}$ \\
\hline
\end{tabular}

\section{Comparative discussion}

To illustrate the effectiveness of the developed algorithm using 2TSFLSs, we solved a numerical example and analyzed the selection of the best alternative, using our developed approach, based on 
2TSFNs. In Table 4, we derived the ranking order of the alternatives by utilizing the developed method. From Table 6, we observed that the ranking orders of the alternatives using the developed method were totally matched with those derived by the Jin et al. [47] method. Therefore, the proposed technique is also validated. Utilizing the proposed technique, the best alternative is $\mathbb{Q}_{1}$, which is the same as the best alternative obtained by the Jin et al. [47] method. Thus, the proposed technique is really prominent, because it can effectively avoid any loss of information which formerly occurred during linguistic information processing. Both numerical and linguistic information are taken into consideration using 2-tuple spherical fuzzy linguistic information, which makes the developed technique more prominent, flexible, and realistic (Table 7).

Table 7. Comparative ranking.

\begin{tabular}{cc}
\hline Operators & Ranking \\
\hline 2TSFLWA & $\mathbb{Q}_{1}>\mathbb{Q}_{3}>\mathbb{Q}_{2}$ \\
2TSFLWG & $\mathbb{Q}_{1}>\mathbb{Q}_{3}>\mathbb{Q}_{2}$ \\
SFLWA [47] & $\mathbb{Q}_{1}>\mathbb{Q}_{3}>\mathbb{Q}_{2}$ \\
SFLWG [47] & $\mathbb{Q}_{1}>\mathbb{Q}_{3}>\mathbb{Q}_{2}$ \\
\hline
\end{tabular}

\section{Conclusions}

In this article, we studied the multi-attribute decision making problem with the 2-tuple spherical fuzzy linguistic environment. First of all we introduced some 2-tuple spherical fuzzy linguistic aggregation operators: 2-tuple spherical fuzzy linguistic weighted average (2TPFLWA), 2-tuple spherical fuzzy linguistic weighted geometric (2TSFLWG), 2-tuple spherical fuzzy linguistic ordered weighted average (2TSFLOWA), 2-tuple spherical fuzzy linguistic ordered weighted geometric (2TSFLOWG), 2-tuple spherical fuzzy linguistic hybrid average (2TSFLHA), and 2-tuple spherical fuzzy linguistic hybrid geometric (2TSFLHG) operators. We also studied some properties of the developed operators. Then, we used these operators and write an algorithm to solve the 2-tuple spherical fuzzy linguistic MADM problem. Lastly, the example of best company selection was given to prove the proposed technique and to establish its practicability and effectiveness. Our future work is to explore the application of 2TSFLNs in many other researches $[46,48,49]$.

Author Contributions: All authors contributed equally in this research paper. All authors have read and agreed to the published version of the manuscript.

Funding: Deanship of Scientific Research (DSR), King Abdulaziz University, Jeddah, under grant No. (DF-765-830-1441).

Acknowledgments: This project was funded by the Deanship of Scientific Research (DSR), King Abdulaziz University, Jeddah, under grant No. (DF-765-830-1441). The authors, therefore, gratefully acknowledge DSR technical and financial support.

Conflicts of Interest: The authors declare no conflict of interest.

\section{References}

1. Zadeh, L.A. Fuzzy sets. Inf. Control 1965, 8, 338-353. [CrossRef]

2. Bellman, R.E.; Zadeh, L.A. Decision-making in a fuzzy environment. Manag. Sci. 1970, 17, B-141-B-164. [CrossRef]

3. Buckley, J.J. Fuzzy decision making with data: Applications to statistics. Fuzzy Sets Syst. 1985, 16, 139-147. [CrossRef]

4. Atnassov, K. Intuitionistic Fuzzy Sets: Theory and Applications; Physica-Verlag: Heidelberg, Germany, 1999; Volume 35.

5. Garg, H. Generalized intuitionistic fuzzy interactive geometric interaction operators using Einstein t-norm and t-conorm and their application to decision making. Comput. Ind. Eng. 2016, 101, 53-69. [CrossRef]

6. Garg, H. Novel intuitionistic fuzzy decision making method based on an improved operation laws and its application. Eng. Appl. Artif. Intell. 2017, 60, 164-174. [CrossRef] 
7. Kaur, G.; Garg, H. Cubic intuitionistic fuzzy aggregation operators. Int. J. Uncertain. Quantif. 2018, 8, 405-427. [CrossRef]

8. Kaur, G.; Garg, H. Generalized cubic intuitionistic fuzzy aggregation operators using t-norm operations and their applications to group decision-making process. Arab. J. Sci. Eng. 2019, 44, 2775-2794. [CrossRef]

9. Garg, H.; Arora, R. Novel scaled prioritized intuitionistic fuzzy soft interaction averaging aggregation operators and their application to multi criteria decision making. Eng. Appl. Artif. Intell. 2018, 71C, 100-112. [CrossRef]

10. Garg, H.; Rani, D. Some generalized complex intuitionistic fuzzy aggregation operators and their application to multi-criteria decision-making process. Arab. J. Sci. Eng. 2019, 44, 2679-2698. [CrossRef]

11. Shen, K.W.; Wang, J.Q. Z-VIKOR method based on a new weighted comprehensive distance measure of z-number and its application. IEEE Trans. Fuzzy Syst. 2018. [CrossRef]

12. Wang, X.K.; Peng, H.G.; Wang, J.Q. Hesitant linguistic intuitionistic fuzzy sets and their application in multi-criteria decision-making problems. Int. J. Uncertain. Quantif. 2018, 8, 321-341. [CrossRef]

13. Garg, H.; Kumar, K. Power Geometric Aggregation Operators Based on Connection Number of Set Pair Analysis Under Intuitionistic Fuzzy Environment. Arab. J. Sci. Eng. 2019, 1-15. [CrossRef]

14. Garg, H.; Rani, D. New generalised Bonferroni mean aggregation operators of complex intuitionistic fuzzy information based on Archimedean t-norm and t-conorm. J. Exp. Theor. Artif. Intell. 2019, 1-29. [CrossRef]

15. Garg, H.; Arora, R. Generalized intuitionistic fuzzy soft power aggregation operator based on t-norm and their application in multicriteria decision-making. Int. J. Intell. Syst. 2019, 34, 215-246. [CrossRef]

16. Yager, R.R.; Abbasov, A.M. Pythagorean membership grades, complex numbers and decision making. Int. J. Intell. Syst. 2013, 28, 436-452. [CrossRef]

17. Yager, R.R. Pythagorean fuzzy subsets. In Proceedings of the 2013 Joint IFSA World Congress and NAFIPS Annual Meeting (IFSA/NAFIPS), Edmonton, AB, Canada, 24-28 June 2013; IEEE: Piscataway, NJ, USA, 2013; pp. 57-61.

18. Rahman, K.; Abdullah, S.; Shakeel, M.; Khan, M.S.A.; Ullah, M. Interval-valued Pythagorean fuzzy geometric aggregation operators and their application to group decision making problem. Cogent Math. Stat. 2017, 4, 1338638. [CrossRef]

19. Liang, D.; Xu, Z. The new extension of TOPSIS method for multiple criteria decision making with hesitant Pythagorean fuzzy sets. Appl. Soft Comput. 2017, 60, 167-179. [CrossRef]

20. Rahman, K.; Ali, A.; Abdullah, S.; Amin, F. Approaches to multi-attribute group decision making based on induced interval-valued Pythagorean fuzzy Einstein aggregation operator. New Math. Nat. Comput. 2018, 14, 343-361. [CrossRef]

21. Garg, H. New exponential operational laws and their aggregation operators for interval-valued Pythagorean fuzzy multi-criteria decision-making. Int. J. Intell. Syst. 2018, 33, 653-683. [CrossRef]

22. Garg, H. Some methods for strategic decision-making problems with immediate probabilities in Pythagorean fuzzy environment. Int. J. Intell. Syst. 2018, 33, 687-712. [CrossRef]

23. Garg, H. Generalized Pythagorean fuzzy geometric aggregation operators using Einstein t-norm and t-conorm for multi-criteria decision-making process. Int. J. Intell. Syst. 2017, 32, 597-630. [CrossRef]

24. Ren, P.; Xu, Z.; Gou, X. Pythagorean fuzzy TODIM approach to multi-criteria decision making. Appl. Soft Comput. 2016, 42, 246-259. [CrossRef]

25. Wei, G. Pythagorean fuzzy interaction aggregation operators and their application to multiple attribute decision making. J. Intell. Fuzzy Syst. 2017, 33, 2119-2132. [CrossRef]

26. Wei, G.; Lu, M. Pythagorean fuzzy power aggregation operators in multiple attribute decision making. Int. J. Intell. Syst. 2018, 33, 169-186. [CrossRef]

27. Xu, Q.; Yu, K.; Zeng, S.; Liu, J. Pythagorean fuzzy induced generalized OWA operator and its application to multi-attribute group decision making. Int. J. Innov. Comput. Inf. Control 2017, 13, 1527-1536.

28. Xue, W.; Xu, Z.; Zhang, X.; Tian, X. Pythagorean fuzzy LINMAP method based on the entropy theory for railway project investment decision making. Int. J. Intell. Syst. 2018, 33, 93-125. [CrossRef]

29. Perez-Domínguez, L.; Rodríguez-Picón, L.A., Alvarado-Iniesta, A.; Luviano Cruz, D.; Xu, Z. MOORA under Pythagorean fuzzy set for multiple criteria decision making. Complexity 2018, 2018, 2602376.

30. Garg, H. A linear programming method based on an improved score function for interval-valued Pythagorean fuzzy numbers and its application to decision-making. Int. J. Uncertain. Fuzziness Knowl.-Based Syst. 2018, 26, 67-80. [CrossRef] 
31. Hamacher, H. Uber logische Verknupfungen unscharfer Aussagen und daren zugeharige Bewertungs-functionene, Progress in Cybernetics and Systems Research; Trappl, R., Klir, G.J., Riccardi, L., Eds.; John Wiley and Sons: New York, NY, USA, 1979; Volume 276, p. 288.

32. Zhou, L.; Zhao, X.; Wei, G. Hesitant fuzzy Hamacher aggregation operators and their application to multiple attribute decision making. J. Intell. Fuzzy Syst. 2014, 26, 2689-2699. [CrossRef]

33. Liu, P. Some Hamacher aggregation operators based on the interval-valued intuitionistic fuzzy numbers and their application to group decision making. IEEE Trans. Fuzzy Syst. 2014, 22, 83-97. [CrossRef]

34. Tan, C.; Yi, W.; Chen, X. Hesitant fuzzy Hamacher aggregation operators for multicriteria decision making. Appl. Soft Comput. 2015, 26, 325-349 [CrossRef]

35. Herrera, F.; Martinez, L. The 2-tuple linguistic computational model: Advantages of its linguistic description, accuracy and consistency. Int. J. Uncertain. Fuzziness Knowl.-Based Syst. 2001, 9 (Suppl. 01), 33-48. [CrossRef]

36. Herrera, F.; Martınez, L.; Sánchez, P.J. Managing non-homogeneous information in group decision making. Eur. Oper. Res. 2005, 166, 115-132. [CrossRef]

37. Wang, W.P. Evaluating new product development performance by fuzzy linguistic computing. Expert Syst. Appl. 2009, 36, 9759-9766. [CrossRef]

38. Wei, G.W. Extension of TOPSIS method for 2-tuple linguistic multiple attribute group decision making with incomplete weight information. Knowl. Inf. Syst. 2010, 25, 623-634. [CrossRef]

39. Chang, K.H.; Wen, T.C. A novel efficient approach for DFMEA combining 2-tuple and the OWA operator. Expert Syst. Appl. 2010, 37, 2362-2370. [CrossRef]

40. Jiang, X.P.; Wei, G.W. Some Bonferroni mean operators with 2-tuple linguistic information and their application to multiple attribute decision making. J. Intell. Fuzzy Syst. 2014, 27, 2153-2162. [CrossRef]

41. Li, C.C.; Dong, Y. Multi-attribute group decision making methods with proportional 2-tuple linguistic assessments and weights. Int. J. Comput. Intell. Syst. 2014, 7, 758-770. [CrossRef]

42. Wang, J.Q.; Wang, D.D.; Zhang, H.Y.; Chen, X.H. Multi-criteria group decision making method based on interval 2-tuple linguistic information and Choquet integral aggregation operators. Soft Comput. 2015, 19, 389-405. [CrossRef]

43. Qin, J.; Liu, X. 2-tuple linguistic Muirhead mean operators for multiple attribute group decision making and its application to supplier selection. Kybernetes 2016, 45, 2-29. [CrossRef]

44. Zhang, W.; Xu, Y.; Wang, H. A consensus reaching model for 2-tuple linguistic multiple attribute group decision making with incomplete weight information. Int. J. Syst. Sci. 2016, 47, 389-405. [CrossRef]

45. Ashraf, S.; Abdullah, S.; Mahmood, T.; Ghani, F.; Mahmood, T. Spherical fuzzy sets and their applications in multi-attribute decision making problems. J. Intell. Fuzzy Syst. 2019, 36, 2829-2844. [CrossRef]

46. Kutlu Gundogdu, F.; Kahraman, C. Spherical fuzzy sets and spherical fuzzy TOPSIS method. J. Intell. Fuzzy Syst. 2019, 36, 337-352. [CrossRef]

47. Jin, H.; Ashraf, S.; Abdullah, S.; Qiyas, M.; Bano, M.; Zeng, S. Linguistic Spherical Fuzzy Aggregation Operators and Their Applications in Multi-Attribute Decision Making Problems. Mathematics 2019, 7, 413. [CrossRef]

48. Ashraf, S.; Abdullah, S.; Mahmood, T. Spherical fuzzy Dombi aggregation operators and their application in group decision making problems. J. Ambient. Intell. Humaniz. Comput. 2019, 1-19. [CrossRef]

49. Ashraf, S.; Abdullah, S.; Aslam, M.; Qiyas, M.; Kutbi, M.A. Spherical fuzzy sets and its representation of spherical fuzzy t-norms and t-conorms. J. Intell. Fuzzy Syst. 2019, 36, 6089-6102. [CrossRef]

50. Herrera, F.; Martínez, L. A 2-tuple fuzzy linguistic representation model for computing with words. IEEE Trans. Fuzzy Syst. 2000, 8, 746-752.

51. Herrera, F.; Martinez, L. An approach for combining linguistic and numerical information based on the 2-tuple fuzzy linguistic representation model in decision-making. Int. J. Uncertainty Fuzziness Knowl. Based Syst. 2000, 8, 539-562. [CrossRef]

52. Yang, M.S.; Hussain, Z. Fuzzy entropy for Pythagorean fuzzy sets with application to multicriterion decision making. Complexity 2018, 2018, 14. [CrossRef]

(C) 2019 by the authors. Licensee MDPI, Basel, Switzerland. This article is an open access article distributed under the terms and conditions of the Creative Commons Attribution (CC BY) license (http:// creativecommons.org/licenses/by/4.0/). 